\title{
A novel targeted co-delivery nanosystem for enhanced ovarian cancer treatment via multidrug resistance reversion and mTOR-mediated signaling pathway
}

Xueqin Wang ${ }^{1,2}$, Tiandi Xiong ${ }^{2}$, Miao Cui ${ }^{2}, \mathrm{Na} \mathrm{Li}^{1,3}$, Qin Li $\mathrm{Li}^{1,3}, \mathrm{Li}_{\text {Zhu }}{ }^{1,3}$, Shaofeng Duan ${ }^{5,6^{*}}$, Yunlong Wang ${ }^{7^{*}}$ and Yuqi Guo $13,4^{*}$

\begin{abstract}
Background: Multidrug resistance (MDR) is the main challenge of successful chemotherapy for ovarian cancer patients, with $50 \%$ to $75 \%$ of ovarian cancer patients eventually relapsed due to it. One of the effective strategies for treating MDR and improving therapeutic efficiency of ovarian cancer is to use nanotechnology-based targeted drug delivery systems. In this study, a novel nano targeted co-delivery system modified by hyaluronic acid (HA) was developed by using gold nanorods coated with functionalized mesoporous silica nanoparticles (HA-PTX/let-7a-GNR@MSN) for combined delivery of hydrophobic chemotherapy drug Paclitaxel (PTX) and lethal-7a (let-7a), a microRNA (miR), to overcome MDR in ovarian cancer. Furthermore, we also analyzed the molecular mechanism of this nanotherapeutic system in the treatment of ovarian cancer.
\end{abstract}

Results: HA-modified nanocomplexes can specifically bind to the CD44 receptor, which is highly expressed in SKOV3/SKOV3 ${ }_{\text {TR }}$ cells, achieving effective cell uptake and 150\% enhancement of tumor site permeability. The nanosystem realized the stable combination and protective transportation of PTX and miRs. Analysis of drug-resistant SKOV $3_{\text {TR }}$ cells and an SKOV3 ${ }_{\text {TR }}$ xenograft model in BALB/c-nude mice showed significant downregulation of P-glycoprotein in heterogeneous tumor sites, PTX release, and subsequent induction of apoptosis. More importantly, this nanosystem could synergistically inhibit the growth of ovarian tumors. Further studies suggest that mTOR-mediated signaling pathways play an important role in reversing drug resistance and inducing apoptosis.

Conclusions: To sum up, these data provide a model for overcoming PTX resistance in ovarian cancer.

Keywords: Ovarian cancer, Multidrug resistance, PTX, miR let-7a, Co-delivery nanosystem

\footnotetext{
*Correspondence: sduan@henu.edu.cn; biowyl@126.com; yuqi-guo@163.

com

${ }_{5}^{1}$ Henan Provincial People's Hospital, Zhengzhou 450003, China

${ }^{5}$ Institute for Innovative Drug Design and Evaluation, School

of Pharmacy, Henan University, Kaifeng 475004, China

${ }^{7}$ Henan Bioengineering Research Center, Zhengzhou 450046, China

Full list of author information is available at the end of the article
}

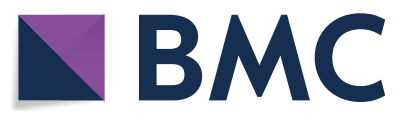

(c) The Author(s) 2021. Open Access This article is licensed under a Creative Commons Attribution 4.0 International License, which permits use, sharing, adaptation, distribution and reproduction in any medium or format, as long as you give appropriate credit to the original author(s) and the source, provide a link to the Creative Commons licence, and indicate if changes were made. The images or other third party material in this article are included in the article's Creative Commons licence, unless indicated otherwise in a credit line to the material. If material is not included in the article's Creative Commons licence and your intended use is not permitted by statutory regulation or exceeds the permitted use, you will need to obtain permission directly from the copyright holder. To view a copy of this licence, visit http://creativecommons.org/licenses/by/4.0/. The Creative Commons Public Domain Dedication waiver (http://creativeco mmons.org/publicdomain/zero/1.0/) applies to the data made available in this article, unless otherwise stated in a credit line to the data. 


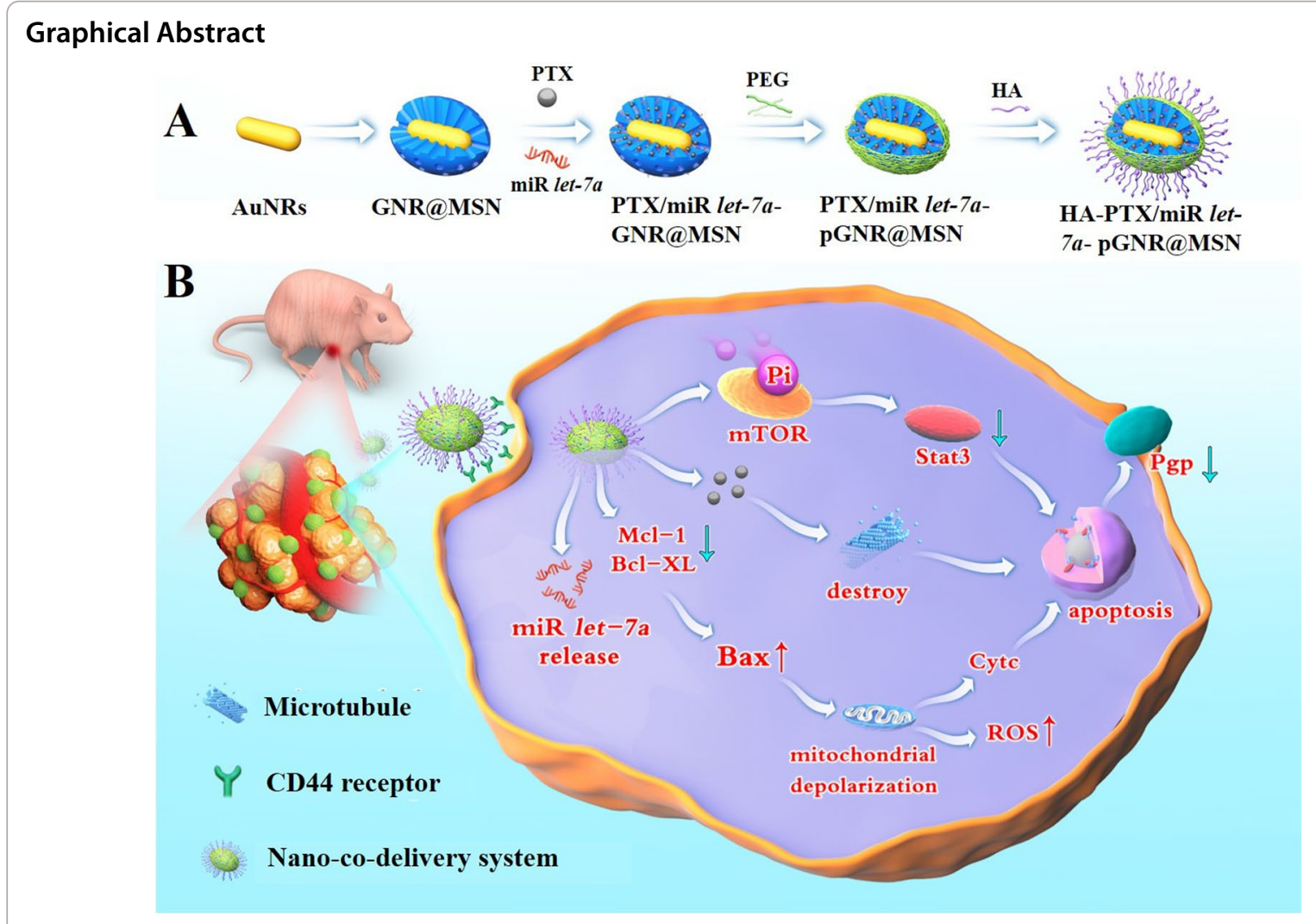

\section{Background}

Ovarian cancer is one of the most serious cancers that harm women's health, with the highest morbidity and mortality among all gynecological malignancies $[1,2]$. The main treatment strategy is surgery combined with chemotherapy based on paclitaxel (PTX), platinum, and other chemotherapy drugs. However, multidrug resistance (MDR) leads to poor effect or even failure of chemotherapy, and $50-75 \%$ of ovarian cancer patients eventually relapse because of MDR [3-6]. The high expression of MDR-related genes in ovarian tumor cells is the main cause of MDR in ovarian cancer [7, 8]. P-glycoprotein (P-gp), a $170 \mathrm{kDa}$ transmembrane glycoprotein (also known as P-170) encoded by the MDR gene family and powered by adenosine triphosphate (ATP), continuously pumps chemotherapeutic drugs out of cancer cells, thereby reducing drug concentrations and cytotoxicity in cancer cells and ultimately leading to MDR. MDR1 inhibitors can inhibit MDR1 expression in cancer cells and reverse $\operatorname{MDR}[9,10]$. However, although MDR1 inhibitors have been used for decades, their clinical effect is not sufficient enough to effectively reverse the MDR in ovarian cancer and improve prognosis. Therefore, it is very important to find a way to reverse MDR in ovarian cancer.

MicroRNAs (miRs) are small, endogenous singlestranded noncoding RNAs with a length of about 20-24 nucleotides. Some miRs, such as lethal 7 (let7) and miR-199a-3p, may have an anti-ovarian cancer effect $[11,12]$. However, these miRs are underexpressed in ovarian cancer cells, promoting cancer growth and anti-apoptosis, and weakening the killing effect of chemotherapy drugs on cancer cells. Therefore, exogenous supplementation of miRs, combined with chemotherapy drugs, shall enhance the anticancer effect and effectively reverse MDR. About $50 \%$ of miRs are located at vulnerable sites of cancer-related genomes. As an oncogene or tumor suppressor gene, miR plays an important role in cancer development and may become an important target for reversing MDR in ovarian cancer $[13,14]$. The let-7 family is closely associated with the development of MDR in ovarian and other cancers [15]. Recent studies have shown that miR let-7 regulates the resistance of ovarian cancer cells to taxol by regulating IMP-1 mediated MDR1 stability [16]. The expression of let-7a in plasma and tissues of patients with ovarian cancer decreased significantly. Therefore, 
we hypothesize that exogenous supplementation of let-7a, combined with chemotherapeutic agents, can enhance the anticancer effect and reverse multidrug resistance in ovarian tumors. However, miR therapy has a potential risk of sequence specificity rather than gene specificity. Therefore, it is of great significance to improve the specificity and targeting of miR for ovarian cancer to improve the therapeutic effect and reduce adverse reactions. In addition, as miRNA is easy to degrade, it is difficult to effectively transfer it to the target. Therefore, it is very significant to develop a strategy to overcome the major hurdles facing the therapeutic miRNA delivery.

In recent years, targeted drug delivery systems based on nanotechnology have been widely used in the prevention, diagnosis, and treatment of various cancers [1719]. Establishing a nanoplatform to co-deliver specific miRs and chemotherapy drugs to overcome MDR and effectively treat ovarian cancer might be a novel strategy. Among various nanobiomaterials, gold nanorods (GNRs) have shown great potential in cancer treatment because of their many advantages. Firstly, GNR preparation is simple to prepare and the particle size can be accurately controlled [20]. Secondly, the large specific surface area (SSA) of GNRs facilitates functional modification and further coupling of targeted ligands, such as receptor-targeted molecules, antibodies, and aptamers. Thirdly, GNRs have good stability and biocompatibility, so they can be used as an ideal carrier for drugs, genes, and nucleic acids [21-23]. However, the application of naked GNRs in drug delivery is limited because of their low drug loading, limited elasticity and cytotoxicity. In contrast, mesoporous silica nanoparticles (MSNs) are attracting increasing interest in gene/drug delivery because of their large pore size, high attraction, and favorable morphological properties [24]. Therefore, we used GNRs as miRNA/PTX delivery vehicle core, and further modified by MSNs with super-large pores which can provide the efficient attachment of miRNA/ PTX, prevent the miRNA degradation, improve drug loading capability and cytotoxicity. The ligand HA was chosen, as HA was tumor-specific for receptor cluster of differentiation 44 (CD44), a membrane-anchored cell surface receptor usually detected in ovarian cancer cells, such as SKOV3 and SKOV3 ${ }_{\mathrm{TR}}$ cells. CD44 has been demonstrated to interact with $\mathrm{HA}$ at the $\mathrm{N}$ terminus of its extracellular domain, and therefore serves as a major cell surface receptor for ovarian cancer $[25,26]$.

In this study, a novel nano targeted co-delivery system modified by hyaluronic acid (HA) was developed employing GNRs coated with functionalized MSNs (GNR@ MSN) (HA-PTX/let-7a-GNR@MSN) to co-deliver miR let-7a and PTX, a hydrophobic chemotherapy drug, to overcome MDR and and enhance the therapeutic efficiency of ovarian cancer treatment (Scheme 1).

\section{Materials and methods \\ Materials}

Gold chloride solution $\left(\mathrm{HAuCl}_{4}\right)$ and tetraethyl orthosilicate (TEOS) were purchased from Aladdin Industrial Co., Ltd. (Shanghai, China); polyethylene glycol $\left(\mathrm{NH}_{2}\right.$-PEG-COOH; molecular weight 3070) from Shanghai ZZBIO Co., Ltd. (Shanghai, China); fetal bovine serum (FBS) and Roswell Park Memorial Institute (RPMI) 1640 culture medium from Gibco (Invitrogen, Carlsbad, CA, USA); HA, acridine orange (AO), fluorescein diacetate (FDA), propidium iodide (PI), Hoechst 33258, 4',6-diamidino-2-phenylindole (DAPI), 3-(4,5-dimethylthiazlo-2-diphenyl-tetrazolium) bromide (MTT), Rhodamine B isothiocyanate (RBITC), PTX, and dimethyl sulfoxide (DMSO) were obtained from SigmaAldrich (St. Louis, MO, USA); an annexin V-fluorescein isothiocyanate (FITC) apoptosis detection kit from Keygen Biotech Co., Ltd. (Nanjing, China); Rhodamine 123 and 2'-7' dichlorofluorescin diacetate (DCFH-DA) from Solarbio Technology Co., Ltd. (Beijing, China). Monoclonal mouse anti-P-gp antibody, Horseradish peroxidase (HRP)-conjugated goat anti-rabbit IgG were obtained from Proteintech Group, Inc. (Chicago, IL, USA); other primary antibodies were purchased from Sigma-Aldrich (St. Louis, MO, USA). Other reagents and chemicals were of analytical grade unless otherwise stated and were purchased from local commercial suppliers. Deionized (DI) water (Milli-Q, Millipore, Bedford, MA, USA) was used to prepare aqueous solutions.

\section{Animals}

About 4-week old male BALB/c-nu mice were obtained from Wuhan Laboratory Animal Co., (Wuhan, China) and were maintained under specific pathogen-free conditions. Animal experiments were conducted according to the guidelines specified by the Animal Care and Use Committee of Zhengzhou University (Zhengzhou, China).

\section{Gold nanorod synthesis}

GNRs were synthesized using the seed growth method [21, 22]. First, gold seeds were synthesized using the chemical reduction method with $\mathrm{HAuCl}_{4}$ as the raw material and $\mathrm{NaBH}_{4}$ as the reducing agent. Next, gold nanoparticles (NPs) were formed in the solution using the gold seed-induced growth method. To prepare a seed solution, $125 \mu \mathrm{L}$ of $1 \% \mathrm{HAuCl}_{4}$ was added to cetyltrimethylammonium bromide (CTAB) $(0.1 \mathrm{M}, 7 \mathrm{~mL})$ under stirring, followed by the addition of $300 \mu \mathrm{L}$ of $0.01 \mathrm{M} \mathrm{NaBH}_{4}$. After the color of the solution changed 

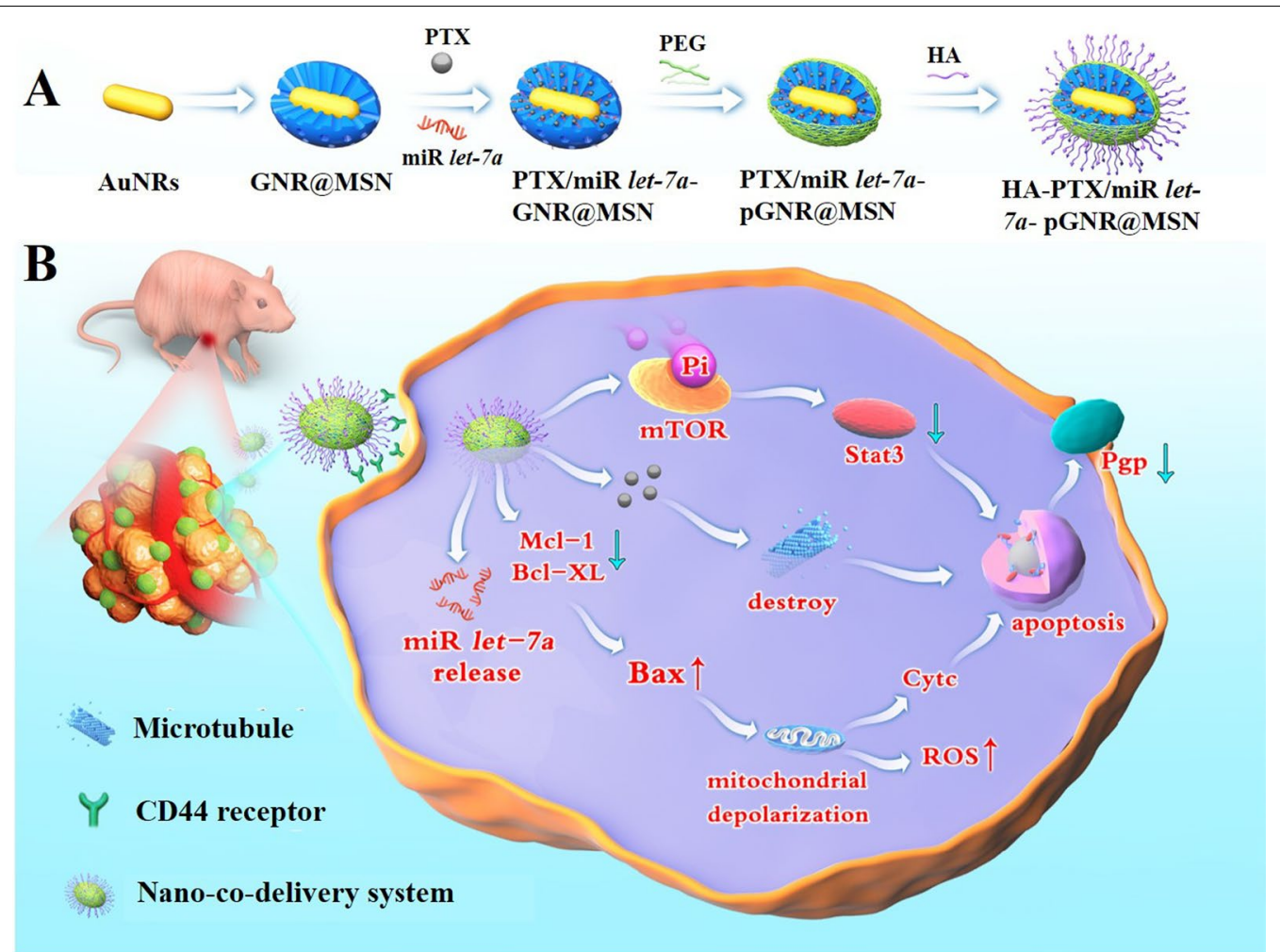

Scheme 1 A Schematic illustration of the synthetic process for the HA-PTX/let-7a-GNR@MSN nano co-deliverysystem. B Serial targeted delivery and therapeutic mechanism of the HA-PTX/let-7a-GNR@MSN co-delivery nanosystem in the ovarian cancer SKOV3/SKOV3 $3_{\text {TR }}$ cell line and tumor tissues. HA, hyaluronic acid; PTX, paclitaxel; miR, microRNA; let-7a, lethal-7a; GNR, gold nanorod; MSN, mesoporous silica nanoparticle; ROS, reactive oxygen species

from golden-yellow to brown, it was kept at $30^{\circ} \mathrm{C}$ for $2 \mathrm{~h}$. To prepare a growth solution, $7 \mathrm{~mL}$ of $0.1 \mathrm{M} \mathrm{CTAB}$ was mixed with $300 \mu \mathrm{L}$ of $1 \% \mathrm{HAuCl}_{4}$ solution, followed by the addition of $10 \mu \mathrm{L}$ of $0.1 \mathrm{M} \mathrm{AgNO}_{3}$ under stirring. Then, $120 \mu \mathrm{L}$ of $0.33 \mathrm{M}$ hydroquinone was added, and the solution quickly became colorless. Finally, $100 \mu \mathrm{L}$ of the seed solution was added, mixed, and allowed to be kept static at $30^{\circ} \mathrm{C}$ for $12 \mathrm{~h}$. The obtained GNRs were collected by centrifugation at $5000 \mathrm{rpm}$, room temperature (RT) for $3 \mathrm{~min}$, dispersed in DI water, and stored at $4{ }^{\circ} \mathrm{C}$ for future use.

\section{GNR@MSN synthesis and amino modification}

The GNRs synthesized were coated with MSNs using the Stober method. Briefly, the $\mathrm{pH}$ of the GNR solution $(0.01 \mathrm{mM}, 10 \mathrm{~mL})$ was adjusted to $10-11$ using $28 \mathrm{wt} \%$ $\mathrm{NH}_{3} \cdot \mathrm{H}_{2} \mathrm{O}$, followed by the addition of $17 \mu \mathrm{L}$ of TEOS every $30 \mathrm{~min}$ four times in total. Subsequently, the reaction was performed at room temperature for $12 \mathrm{~h}$. Then, the obtained GNR@MSN was rinsed by centrifugation with DI water and methanol at 5000 RPM at RT for 5 min, respectively.
For amino modification of GNR@MSN, the nanoparticles were dispersed in the mixture of $15 \mu \mathrm{L}$ of 3-aminopropyltriethoxysilane (APTES) and $85 \mu \mathrm{L}$ of methanol, and then refluxed at $75{ }^{\circ} \mathrm{C}$ for $6 \mathrm{~h}$. Finally, the obtained GNR@MSN-NH $\mathrm{N}_{2}$ were washed thrice with hot methanol by centrifugation at $5000 \mathrm{rpm}$ for $10 \mathrm{~min}[27,28]$.

\section{PTX and miR let-7a loading}

PTX and miR let-7a were loaded onto GNR@MSN-NH ${ }_{2}$ through pore adsorption of mesoporous silica, where the mass ratio of PTX to GNR@MSN-NH $\mathrm{N}_{2}$ was 1:1. Briefly, PTX was dissolved in $1 \mathrm{~mL}$ of DMSO and added to a solution of GNR@MSN-NH$H_{2}$. Then, the reaction proceeded overnight under continuous shaking [27]. Next, the obtained PTX-GNR@MSN were collected by centrifugation at 10,000 rpm for $10 \mathrm{~min}$ and dried under vacuum at $45{ }^{\circ} \mathrm{C}$ overnight. Subsequently, $0.25 \mathrm{mg}$ PTXGNR@MSN was dispersed in $40 \mu \mathrm{L}$ of ethanol, followed by the addition of $10 \mu \mathrm{L}$ of $4 \mathrm{M} \mathrm{HCl}$ and miR let- $7 a$ $(20 \mu \mathrm{mol} / \mathrm{L})$ for $2 \mathrm{~h}$, the obtained PTX/miR let-7a-GNR@ MSN were centrifuged at 10,000 rpm for $10 \mathrm{~min}$ [29]. The loading degrees of PTX and miR let-7a were determined 
by high-performance liquid chromatography (HPLC) and agarose gel electrophoresis, respectively, as shown below:

$$
\begin{aligned}
& \text { Loading content }(\%)=\frac{W_{\mathrm{t}}}{W_{\mathrm{s}}} \times 100 \%, \\
& \text { Encapsulation efficiency }(\%)=\frac{W_{\mathrm{t}}}{W_{0}} \times 100 \%,
\end{aligned}
$$

where $W_{\mathrm{t}}$ is the weight of PTX in NPs, $W_{\mathrm{s}}$ is the weight of NPs, and $W_{0}$ is the initial weight of PTX in the system.

\section{Fabrication of PEG-modified GNR@MSN}

PEG-modified GNR@MSN (pGNR@MSN) were fabricated using 1-ethyl-3-(3-dimethyllaminopro-pyl) carbodiimide hydrochloride/ $N$-hydroxysuccinimide (EDC/NHS) coupling method. Briefly, $10 \mathrm{mg}$ of $\mathrm{NH}_{2}$-PEG-COOH was dissolved in $8 \mathrm{~mL}$ of DMSO, followed by the addition of $38 \mathrm{mg}$ of NHS and $68 \mathrm{mg}$ of EDC dissolved in $2 \mathrm{~mL}$ of 2 - $(N$-morpholino) ethanesulfonic acid (MES) buffer ( $\mathrm{pH}$ 6.0). After the activation reaction proceeded at room temperature (RT) for $24 \mathrm{~h}$, $10 \mathrm{mg}$ GNR@MSN-NH $\mathrm{H}_{2}$ was added and the reaction was kept at RT for another $24 \mathrm{~h}$. Finally, the nanoparticles obtained by centrifuging the reaction mixture at $10,000 \mathrm{rpm}$ for $10 \mathrm{~min}$ were washed with deionized water to remove excess PEG to obtain pGNR@MSN, which were dried under vacuum at $45{ }^{\circ} \mathrm{C}$ overnight and stored at $4{ }^{\circ} \mathrm{C}$ until use.

\section{Preparation of HA-modified nanocomposites}

$10 \mathrm{mg}$ of HA was added to MES buffer (to $\mathrm{pH} 6.0$ ) containing $5 \mathrm{~mL}$ EDC/NHS $(2 \mathrm{mg} / \mathrm{mL})$ for activation $1.5 \mathrm{~h}$ and then the $\mathrm{pH}$ of the solution was adjusted to 8.3 using Tris buffer. After the activation reaction proceeded for 12 h, 10 mg of pGNR@MSN were added and the reaction was continued at RT for $24 \mathrm{~h}$. Finally, the nanoparticles obtained by centrifuging the reaction mixture at 10,000 RPM for 10 min were washed with methanol and deionized water to remove the impurities to obtain HApGNR@MSN, which were dried under vacuum $45{ }^{\circ} \mathrm{C}$ overnight and stored at $4{ }^{\circ} \mathrm{C}$ until use.

\section{Characterization}

The morphology of the as-prepared pGNR@MSN was characterized employing high-angle annular dark-field scanning transmission electron microscopy (HAADFSTEM) and high-resolution transmission electron microscopy (HRTEM) using a JEM-2100 analytical electron microscope (JEOL Ltd., Tokyo, Japan). The crystal structure of the GNRs was measured using X-ray diffraction (XRD; X'Pert PRO MPD, Hol-land Panalytical) with a monochromatic $\mathrm{X}$-ray beam and nickel-filtered $\mathrm{Cu}$
Ka radiation and the Fourier transform infrared (FT-IR) spectra of the pGNR@MSN were recorded using a FT-IR spectrometer (Nicolet IS50-Continuum; Thermo Fisher Scientific, Waltham, MA, USA). In addition, the $\mathrm{N}_{2}$ adsorption-desorption isothermal curve was recorded using the TriStar II 3020 system (Micromeritics, USA), and the zeta potential and particle size of the nanoparticles were determined using a Zetasizer Nano ZS90 analyzer (Malvern Panalytical, Malvern, Netherland).

\section{Cell culture}

The ovarian cancer SKOV3 cell line was purchased from the Shanghai Cell Bank of the Chinese Academy of Sciences (Shanghai, China). SKOV3 cells were cultured in RPMI 1640 medium containing 10\% FBS, $100 \mu \mathrm{g} / \mathrm{mL}$ of penicillin, and $100 \mu \mathrm{g} / \mathrm{mL}$ of streptomycin in a $5 \% \mathrm{CO}_{2}$ humidified atmosphere at $37{ }^{\circ} \mathrm{C}$. The $\mathrm{SKOV} 3_{\mathrm{TR}}$ cell line (PTX-resistant SKOV3 cells) was constructed and maintained in RPMI 1640 medium containing $10 \mu \mathrm{L}$ of PTX $(2 \mathrm{mg} / \mathrm{mL})$.

\section{Hemolysis assay}

The blood compatibility of HA-pGNR@MSN was evaluated by hemolysis assay. Briefly, fresh human blood was centrifuged at $3000 \mathrm{rpm}$ for $15 \mathrm{~min}$ and the supernatant was removed, followed by washing with $0.9 \%$ saline solution to obtain red blood cells (RBCs). Next, a proper amount of $0.9 \%$ saline was added to the RBCs to prepare a suspension contaiing $2 \% \mathrm{RBC}$ and $1 \mathrm{~mL}$ of such suspension was then added to the solution of HA-pGNR@MSN at different concentrations $(50-400 \mu \mathrm{g} / \mathrm{mL})$ with saline and DI water as negative and positive controls, respectively. The samples were stabilized at RT for $2 \mathrm{~h}$ and then centrifuged at $3000 \mathrm{rpm}$. Finally, the supernatant was collected and measured absorbance at the wavelength of $540 \mathrm{~nm}$ to obtain the image of the sample and calculate the hemolysis rate.

\section{in vitro cytotoxicity assay}

The cytotoxicity of HA-pGNR@MSN was detected using 3-(4,5-dimethylthiazol-2-yl)-2,5- diphenyltetrazolium bromide (MTT) assay. Briefly, SKOV3 cells were seeded on a 96-well plate at a density of 3000 cells/well and incubated for $12 \mathrm{~h}$. Next, a series of HA-pGNR@MSN solutions at different concentrations $(0,25,50,100,200$, and $400 \mu \mathrm{g} / \mathrm{mL}$ ) were added to the 96-well plate. After incubation at $37{ }^{\circ} \mathrm{C}$ for $24 \mathrm{~h}$, the medium was removed and $0.5 \mathrm{mg} / \mathrm{mL}$ of MTT solution ( $200 \mu \mathrm{L} /$ well), was added, followed by incubation for another $4 \mathrm{~h}$. Subsequently, DMSO was added $(150 \mu \mathrm{L} /$ well $)$ and the mixture was vortexed. Finally, the absorbance of the sample was measured at the wavelength of $570 \mathrm{~nm}$ using a microplate 
spectrophotometer (BioTek Instruments Inc., Winooski, VT, USA) [30].

\section{RBITC-labeled nanocomposites for cell uptake analysis} Cell uptake analysis was performed using fluorescent RBITC-labeled nanocomposites. Briefly, HA-pGNR@ MSN and pGNR@MSN were labeled using fluorescent RBITC via the amino groups on the surface of the nanoparticles [31]. Next, SKOV3 cells were seeded on a 24-well plate and incubated for $48 \mathrm{~h}$, followed by the addition of fluorescent RBITC-labeled HA-pGNR@MSN and pGNR@MSN, followed by incubatiton in the dark for another $4 \mathrm{~h}$. The cells were then rinsed thrice with PBS and photographed under an inverted EclipseTE2000-U fluorescence microscope (Nikon, Japan). RNA transfection efficiency was detected employing carboxyfluorescein (FAM)-labeled miR let-7a, whose uptake by $\mathrm{SKOV}_{\mathrm{TR}}$ cells was performed as mentioned earlier.

\section{Analysis of antiproliferation effects}

The anti-proliferation ability of various therapeutic nanocomposites was examined using MTT assay and cell viability was assessed using an FDA and PI double staining method [32]. Briefly, SKOV3 and SKOV3 ${ }_{\mathrm{TR}}$ cells treated with various therapeutic nanocomposites were washed with PBS and incubated with $1 \mu \mathrm{g} / \mathrm{mL}$ of FDA and $20 \mu \mathrm{g} /$ $\mathrm{mL}$ of PI for $5 \mathrm{~min}$. The cells were then washed with PBS and photographed under an inverted Eclipse TE2000-U fluorescence microscope.

\section{Apoptosis assay}

Hoechst 33258 was used to detect the apoptosis of SKOV3 and SKOV3 $3_{\mathrm{TR}}$ cells treated with various therapeutic nanocomposites [33]. Briefly, SKOV3 and SKOV3 ${ }_{\mathrm{TR}}$ cells were cultured on a 24-well plate for $12 \mathrm{~h}$, followed by the addition of HA-miR let-7a-GNR@MSN, HA-PTX-GNR@MSN, PTX/miR let-7a-GNR@MSN, and HA-PTX/miR let-7a-GNR@MSN, respectively. After treatment for $24 \mathrm{~h}$, the drug and the original culture solution were removed and the treated cells were rinsed with PBS once. Next, $500 \mu \mathrm{L}$ of $0.5 \mathrm{mg} / \mathrm{mL}$ Hoechst 33258 staining solution was added to each well, followed by incubation in the dark for $20 \mathrm{~min}$. The cells were then washed with PBS twice to remove the unreacted staining solution and observed under an inverted Eclipse TE2000$\mathrm{U}$ fluorescence microscope.

Annexin V-FITC/PI double-staining assay was used to detect the apoptosis of treated SKOV3 and SKOV3 ${ }_{\mathrm{TR}}$ cells. Briefly, the treated SKOV3 and $\mathrm{SKOV} 3_{\mathrm{TR}}$ cells were digested using $0.25 \%$ trypsin and then centrifuged at $1000 \mathrm{rpm}$ for $5 \mathrm{~min}$ for collection. Next, the cells collected were resuspended in $500 \mu \mathrm{L}$ of PBS, followed by the addition of $5 \mu \mathrm{L}$ of annexin V-FITC and PI, respectively.
Finally, the mixture was incubated in the dark at RT for $10 \mathrm{~min}$ and then the cells were analyzed using FACS Calibur flow cytometry (BD Biosciences, San Jose, CA, USA) and Cell-Quest software (BD Biosciences).

\section{Mitochondrial membrane potential detection}

Mitochondrial membrane potential (MMP) was detected by Rhodamine 123 staining. Briefly, SKOV3 and SKOV3 ${ }_{\mathrm{TR}}$ cells were treated with various therapeutic nanocomposites for $24 \mathrm{~h}$, washed with PBS twice, stained with $50 \mu \mathrm{g} / \mathrm{mL}$ of Rhodamine 123 , and incubated in the dark for $30 \mathrm{~min}$. Next, the cells were again washed with PBS twice and photographed under an inverted EclipseTE2000-U fluorescence microscope.

\section{Analysis of reactive oxygen species}

SKOV3 and SKOV3 ${ }_{\mathrm{TR}}$ cells treated with various therapeutic nanocomposites were washed twice with PBS, treated with $50 \mu \mathrm{M}$ DCFH-DA in FBS-free medium, and incubated in the dark for $15 \mathrm{~min}$. Next, the cells were again washed with PBS twice, and reactive oxygen species (ROS) were analyzed using FACS Calibur flow cytometry (BD Biosciences, San Jose, CA, USA) and CellQuest software (BD Biosciences).

\section{Acridine orange staining}

SKOV3 and SKOV3 ${ }_{\mathrm{TR}}$ cells treated with various therapeutic nanocomposites for $24 \mathrm{~h}$ were washed with $\mathrm{PBS}$ twice and then resuspended in $300 \mu \mathrm{L}$ of PBS. Next, $300 \mu \mathrm{L}$ of $0.01 \% \mathrm{AO}$ staining solution was added, followed by incubation the dark at RT for $15 \mathrm{~min}$. Next, the cells were washed with PBS twice and photographed under an inverted fluorescence Eclipse TE2000-U microscope.

\section{Western blotting}

SKOV3 and SKOV3 ${ }_{\mathrm{TR}}$ cells treated with various therapeutic nanocomposites were lysed in lysis buffer (50 mM Tris- $\mathrm{HCl}$ [pH 7.4], $150 \mathrm{mM} \mathrm{NaCl}, 1 \% \mathrm{NP}-40$, $0.5 \%$ sodium dexoycholate, $0.1 \%$ sodium dodecyl sulfate [SDS], $2 \mathrm{mM}$ phenylmethylsulfonyl fluoride [PMSF]) and then boiled for $5 \mathrm{~min}$ in a metal bath at $100{ }^{\circ} \mathrm{C}$. Next, the total protein extracts $(10 \mu \mathrm{g})$ were separated employing $12 \%$ sodium dodecyl sulfate-polyacrylamide gel electrophoresis (SDS-PAGE) and then transferred onto a polyvinylidene difluoride (PVDF) membrane (Merck Millipore, Burlington, MA, USA) blocked with $8 \%(\mathrm{w} / \mathrm{v})$ nonfat milk in $0.05 \%$ phosphate-buffered saline-Tween 20 (PBST) buffer for $1 \mathrm{~h}$. The PVDF membrane was washed with PBST buffer and then incubated overnight with primary antibodies (1:5000 or 1:10,000 in PBST) at $4{ }^{\circ} \mathrm{C}$ and appropriate horseradish peroxidase (HRP)-conjugated secondary antibodies $(1: 10,000$ or $1: 20,000)$ for $1 \mathrm{~h}$ at 
RT. Finally, immunoreactive bands were developed using Pierce ECL Western Blotting Substrate (Thermo Fisher Scientific), and the relative protein quantity was normalized to the level of glyceraldehyde 3-phosphate dehydrogenase (GADPH).

\section{Establishment of SKOV ${ }_{\mathrm{TR}}$ tumor xenograft model}

Four-week-old male BALB/c-nu mice were obtained from Wuhan Laboratory Animal Co., (Wuhan, China) and maintained under the specific pathogen-free (SPF) condition. SKOV $3_{\mathrm{TR}}$ cells at the logarithmic growth stage were used to prepare $100 \mu \mathrm{L}$ cell suspension (density: $8 \times 10^{6}$ cells). The suspension was inoculated subcutaneously on the dorsal side of mice. The health status and behavior of the mice were observed every day and the tumor volume and body weight were recorded every 2 days. The tumor volume was calculated as follows:

$$
V=1 / 2 \times a \times b^{2},
$$

where $a$ and $b$ are the long and short diameters of the tumor, respectively. The tumor suppression rate was calculated as follows:

$$
R(\%)=\left(1-V_{\mathrm{t}} / V_{0}\right) \times 100 \%,
$$

where $V_{\mathrm{t}}$ and $V_{0}$ are the tumor volume of the treatment and the control group, respectively. When the average tumor volume reached $30 \mathrm{~mm}^{3}$, various therapeutic nanocomposites prepared for drug test were injected.

\section{Drug intervention and histopathological analysis}

In the successfully established $\mathrm{SKOV}_{\mathrm{TR}}$ tumor xenograft model, the BALB/c-nu mice were randomly divided into five groups and treated with various therapeutic nanocomposites: HA-miR let-7a-pGNR@MSN, HAPTX-pGNR@MSN, PTX/miR let-7a-pGNR@MSN, and HA-PTX/miR let-7a-pGNR@MSN, with 0.9\% saline as control. Intratumoral injection was performed using the whole constructive nanocomposites at a rate of $15 \mathrm{mg} / \mathrm{kg}$ $(200 \mu \mathrm{L})$ every 2 days $[34,35]$. After treatment, the mice were euthanized, and the tumors and main organs (liver, heart, lungs, kidneys, and spleen) were collected for histopathological analysis by hematoxylin and eosin (H\&E) staining, terminal deoxynucleotidyl transferase dUTP nick end labeling (TUNEL) staining, and antigen Ki-67 staining. Finally, the corresponding tumor sites were analyzed by western blotting to detect the expression of P-gp.

\section{Statistical analysis}

All the data were presented as mean \pm standard deviation (SD). Student's $t$-test was used for statistical comparison. ${ }^{*} \mathrm{p}<0.05$ was considered significant, ${ }^{*} \mathrm{p}<0.01$ was considered moderately significant) and ${ }^{* * *} \mathrm{p}<0.001$ was considered highly significant.

\section{Results and discussion}

\section{Synthesis and characterization of HA-pGNR@MSN}

Herein, we report a novel HA-modified targeted nano drug delivery system that uses functionalized mesoporous silica nanoparticle-coated gold nanorods (HA-GNR@MSN) to co-deliver PTX and miR let-7a to overcome MDR in ovarian cancer.

The synthesis procedure of the targeted nanosystem is shown in Scheme 1, which includes: (i) synthesis of GNRs utilizing improved seed growth method; (ii) encapsulation of GNR with mesoporous silicon shells with large aperture to to load PTX and miR let-7a by aperture adsorption and electrostatic shielding method; (iii) amine modification with APTES; (iv) loading PTX and miR let-7a into silicon pores; (v) modification with $\mathrm{NH}_{2}$-PEG-COOH (to ensure the blood circulation time and subsequent modification ability of the nanoparticles); and (vi) conjugation of this nanocomposite with HA via EDC/NHS to forme a specifically targeted Nano delivery system (HA-PTX/let-7a-GNR@MSN) to effectively enhance ovarian cancer treatment through multidrug resistance reversal.

The as-prepared nanoparticle complexes were characterized subsequently. Transmission electron microscopy (TEM) showed that the average length and width of the prepared GNRs were $75 \pm 5$ and $5 \pm 5 \mathrm{~nm}$, respectively ( 7:1 aspect ratio) (Fig. 1A). Further measurement of the GNRs by HRTEM showed clearly visible bright and dark stripes of the lattice spacing (Fig. 1B). In addition, TEM of the obtained GNR@MSN core-shell NPs indicated that the GNRs were successfully coated by mesoporous silicon, and the average diameter of GNR@MSN was $135 \pm 5 \mathrm{~nm}$ (Fig. 1C, D). In HAADF mode, analysis of GNR@MSN using a dark-field detector and energydispersive X-ray spectroscopy (EDS) element mapping showed that the GNRs were mainly distributed in the brighter region. In addition, the two elements $(\mathrm{Au}, \mathrm{Si})$ in GNR@MSN were also distributed in the brighter region, as expected (Fig. 1E). The diffraction peaks of the GNRs appeared at $2 \theta$ of $38.2^{\circ}, 44.4^{\circ}, 64.6^{\circ}, 77.6^{\circ}$, and $81.7^{\circ}$, which were correlated with the lattice planes (111), (200), (220), (311), and (222) of Au, respectively (Fig. 1F).

The detailed structure of HA-modified nanocomposites was shown in Fig. 2A. The zeta potential changes of various therapeutic nanocomposites $(+13.00,-6.94$, $+21.70,-3.55$, and $-37.33 \mathrm{mv}$ ) confirmed the successful modification of each component (Fig. 2B). The hydrodynamic diameter distributions of the prepared NPs were 255.7, 398.7, 488.9, 802.2, and $929.7 \mathrm{~nm}$ respectively, corresponding to GNRs, GNR@MSN, GNR@MSN-NH ${ }_{2}$, 

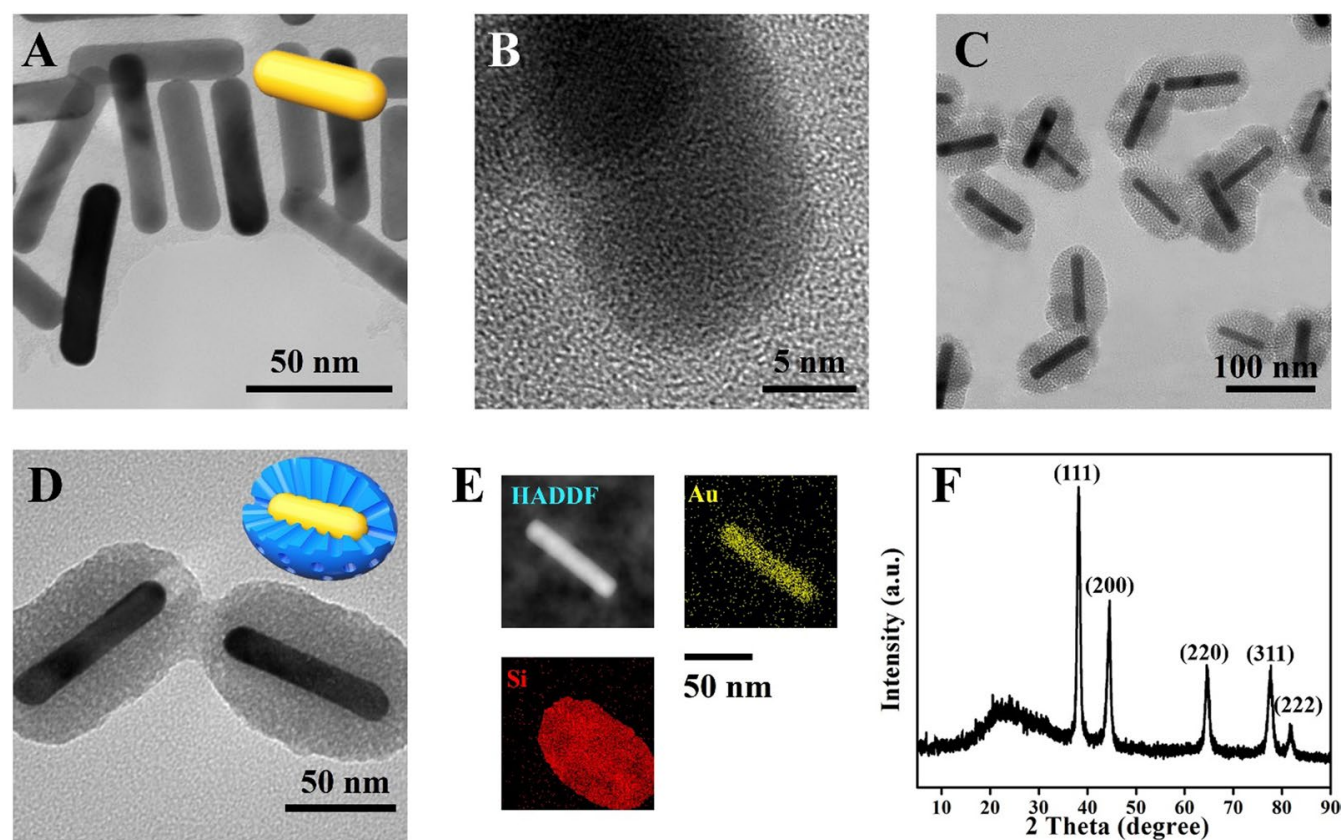

Fig. 1 Characterization of GNR and GNR@MSN. A TEM and $\mathbf{B}$ HRTEM images of GNRS, C TEM, D partial enlarged TEM, E HAADF and EDS mapping images of GNR@MSN.F XRD patterns of GNRs. GNRs, gold nanorods; GNR@MSN, mesoporous silica nanoparticle-coated gold nanorods; TEM, transmission electron microscopy; HRTEM, high-resolution transmission electron microscopy; HAADF, high-angle annular dark-field; EDS, energy-dispersive $\mathrm{X}$-ray spectroscopy; $\mathrm{XRD}, \mathrm{X}$-ray diffraction

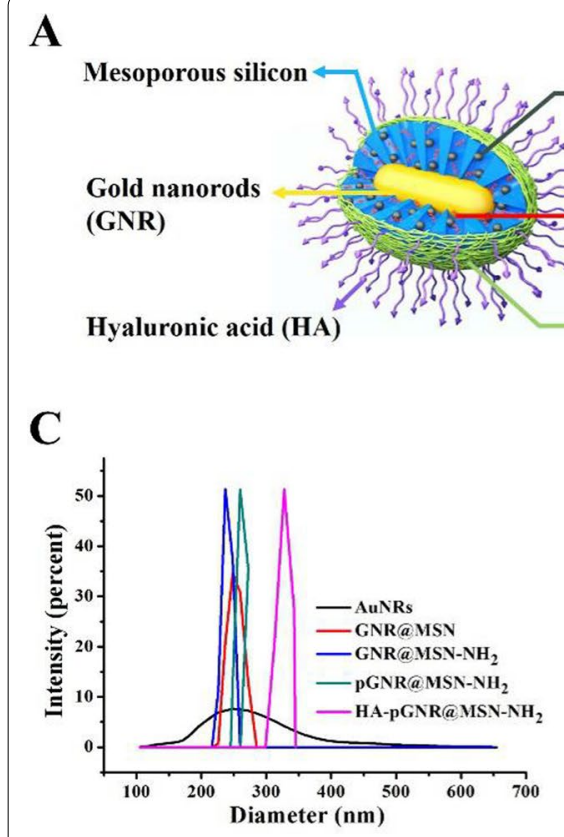

Chemotherapy drugs PTX

Gene drugs miR let-7a

PEG

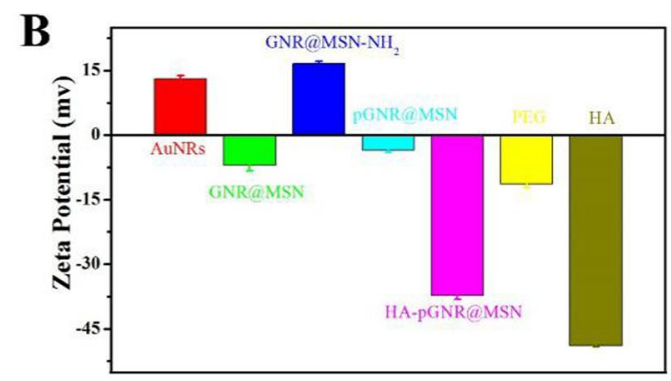

D

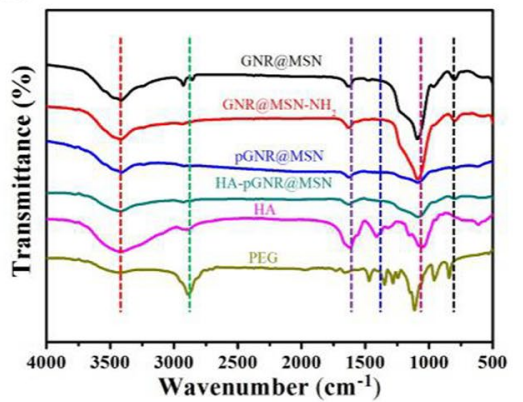

$\mathbf{E}$

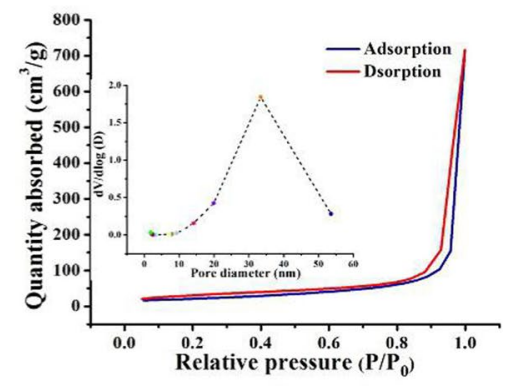

Fig. 2 Characterization of HA-pGNR@MSN. A Structure of HA-pGNR@MSN. B Zeta potential assay of GNRs and various therapeutic nanocomposites. C Diameter distribution of GNRs and various therapeutic nanocomposites. D FT-IR spectra of GNRs, GNR@MSN, GNR@MSN-NH 2 , pGNR@MSN, and HA-pGNR@MSN.E N 2 absorption-desorption isotherm of GNR@MSN and corresponding pore size distribution curve (inset). HA, hyaluronic acid; pGNR, polyethylene glycol-modified gold nanorod; MSN, mesoporous silica nanoparticle; FT-IR, Fourier transform infrared 
pGNR@MSN, and HA-pGNR@MSN (Fig. 2C). The obtained hydrated particle size data may be caused by polyhydroxy polymer compounds such as polyethylene glycol (PEG) and HA.

Further, FT-IR spectroscopy was used to detect the structure of the nanocomposites. The FT-IR spectrum of GNR@MSN-NH $\mathrm{N}_{2}$ contained signals associated with $\mathrm{N}-\mathrm{H}$ $\left(1560 \mathrm{~cm}^{-1}\right)$, indicating that the amination of GNR@ MSN was successful. Owing to the modification of the MMSN surface with $\mathrm{HA}$, the characteristic bands of amide bond were identified at the amide $\mathrm{N}-\mathrm{H}$ stretching $\left(3680 \mathrm{~cm}^{-1}\right)$ and amide $\mathrm{C}=\mathrm{O}$ stretching $\left(1690 \mathrm{~cm}^{-1}\right)$. In addition, the vibrational absorption peaks of $\mathrm{Si}-\mathrm{O}-\mathrm{Si}$ in GNR@MSN appear at $1081 \mathrm{~cm}^{-1}$ and $798 \mathrm{~cm}^{-1}$. The pore size and SSA of GNR@MSN were determined by the $\mathrm{N}_{2}$ absorption-desorption technique using BarrettJoyner-Halenda (BJH) and Brunauer-Emmett-Teller (BET) methods. The SSA was high $\left(77.00 \mathrm{~m}^{2} / \mathrm{g}\right)$ and the average pore size was $34.22 \mathrm{~nm}$ (Fig. 2E), indicating that the pores of mesoporous silica could effectively adsorb PTX and miR let-7a.
Evaluation of biosafety, stability and fluorescent properties of nanocomposites

Low cytotoxicity is crucial to construct HA-pGNR@ MSN nano co-delivery system. First, the cytotoxicity of HA-pGNR@MSN at different concentrations was assessed. SKOV3 cells showed no significant cytotoxicity after treatment with $0,25,50,100,200$, and $400 \mu \mathrm{g} / \mathrm{mL}$ of HA-pGNR@MSN for 24h (Fig. 3A).

Hemocompatibility is another vital issue in bloodcontacting applications of NPs [28]. Therefore, the hemolysis of HA-pGNR@MSN before use for gene/drug delivery was examined. Although the concentration of HA-pGNR@MSN reached $400 \mu \mathrm{g} / \mathrm{mL}$, the hemolysis rate was only $4.85 \%(<5 \%)$, (Fig. $3 \mathrm{~B}, \mathrm{C})$, indicating that HApGNR@MSN could be used for subsequent in vivo and in vitro experiments. The stability of the prepared HApGNR@MSN in different media including 1640 medium, PBS (pH 7.4), and DI water was presented in Additional file 1: Fig. S2A, indicating the composites had excellent stability in different media. Besides, RBITC-labeled NPs showed strong red fluorescence, indicating that they

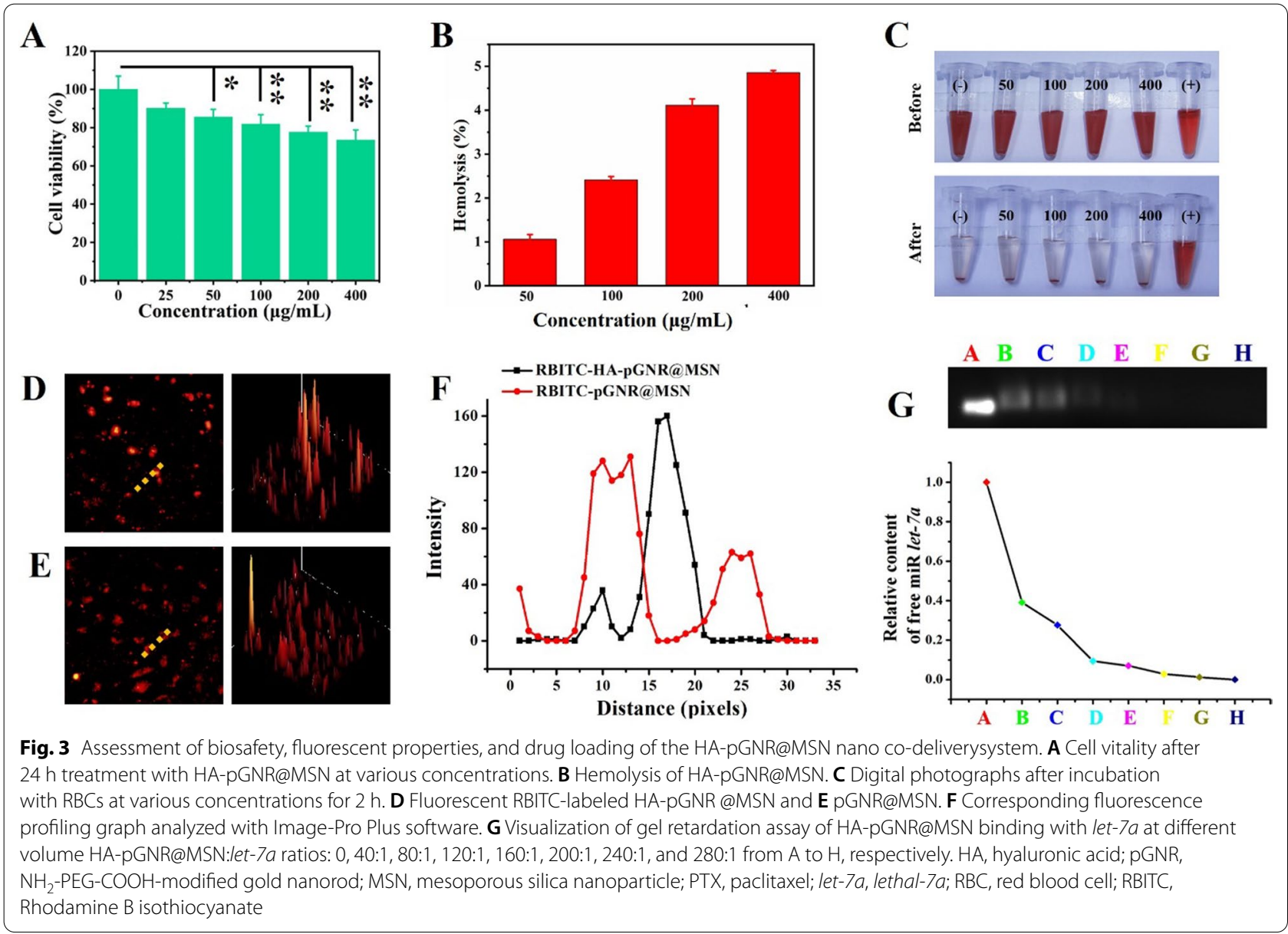


could be effectively used subsequent cell uptake analysis (Fig. 3D-F).

\section{Drug loading and release of the HA-PTX/let-7a-GNR@MSN nano co-delivery system}

Hydrophobic drugs are difficult to bind to NPs [26]. In addition, as RNA is easy to degrade, it is difficult to effectively transfer it to the target [27]. Therefore, GNR was modified by MSN with super-large pores to prepare PGNR for drug adsorption. The PEG of pGNR@MSN can prolong the blood circulation time of a drug. In addition, the targeted ligand HA can specifically distinguish the CD44 receptor, which is highly expressed in ovarian cancers SKOV3/SKOV $3_{\mathrm{TR}}$. Therefore, the administration efficiency can be improved and the therapeutic effect can be further improved.

The loading degree of PTX was determined by HPLC. According to the peak area and standard curve equation of PTX in Additional file 1: Fig. S1A, B, the standard equation was $y=-7.8 \times 10^{5}+5.1 \times 10^{5} x\left(r^{2}=0.9909\right)$ and the drug loading was $3.82 \%$.

In addition, the controlled release of PTX from HAPTX/ miR let-7a-GNR@MSN was evaluated in the PBS solution with representative $\mathrm{pH}$ including $(\mathrm{pH} 5.0$ and 7.4) (Additional file 1: Fig. S2B), and the results showed that the release of free PTX much faster than loading onto HA-PTX/miR let-7a-GNR@MSN at pH 5.0 and 7.4, approximately $90.7 \%(\mathrm{pH} 5.0)$ and $78.6 \%(\mathrm{pH} 7.4)$ was released within $12 \mathrm{~h}, 64.3$ and $43.7 \%$ of PTX was released from HA-PTX/miR let-7a-GNR@MSN during the $72 \mathrm{~h}$ at $\mathrm{pH} 5.0$ and $\mathrm{pH}$ 7.4. The sustained release of PTX from HA-PTX/miR let-7a-GNR@MSN could be beneficial for enhancing long-term antitumor efficiency and improving drug accumulation at a targeted site. These data also show the release of PTX is more rapidly in the mildly acidic microenvironment in the tumor area than in normal tissue or blood. PTX, as a classical clinical anti-tumor drug, can disbalance the synthesis and depolymerization of tubulin, induce and promote tubulin polymerization and microtubule assembly, and trigger cell apoptosis, thereby effectively preventing the proliferation of ovarian cancer cells.

In order to obtain the optimal binding ratio of miR let$7 a$ and nanocarrier, agarose gel electrophoresis assay was performed. The final concentration of miR let-7a was $20 \mu \mathrm{M}$, and a series of proportional gradiations of GNR@ MSN- $\mathrm{NH}_{2}$ and miR let-7a were set as 40:1, 80:1, 120:1, 160:1, 200:1, 240:1, and 280:1 (w/w). Next, gel block was used to determine the optimal ratio. At the ratio of 200:1, no free let-7a was found in the supernatant (Fig. 3G), indicating that $\mathrm{miR}$ let-7a was completely bound to
GNR@MSN- $\mathrm{NH}_{2}$ at this ratio. In the following experiment, the ratio of $200 \mu \mathrm{g}: 1 \mu \mathrm{g}$ (GNR@MSN-NH 2 :miR let-7a) was used for subsequent drug intervention experiments.

For cellular uptake assay, RBITC-labeled NPs can be effectively endocytosed via targeted HA, and they effectively recognize and bind to the CD44 receptor, which is highly expressed in SKOV3/SKOV3 ${ }_{\mathrm{TR}}$ cells, thus improving cell uptake efficiency. The cellular uptake efficiency of HA-pGNR@MSN increased 300\% times than pGNR@ MSN (Additional file 1: Fig. S3A, B). Fluorescent doublelabeled HA- ${ }^{\mathrm{FAM}}$ miR let-7a- ${ }^{\text {RBITC }}$ pGNR@MSN was used to further confirm the transfection effeciency of the NPs after co-incubation with $\mathrm{SKOV}_{\mathrm{TR}}$ cells for 6,12 , and $24 \mathrm{~h}$. The result demonstrated that the targeted HApGNR@MSN more effectively delivered miR let-7a to SKOV3/SKOV $3_{\mathrm{TR}}$ cells, and the maximum transfection rate was achieved $12 \mathrm{~h}$ post treatment (Additional file 1: Fig. S3C).

\section{Antiproliferative and therapeutic effects of nanocomposites in vitro}

MTT assay was used to evaluate the anti-proliferative ability of the therapeutic nanocomposites. Compared to PTX/miR let-7a-GNR@MSN, HA-PTX/miR let7a-GNR@MSN showed a better therapeutic effect on SKOV3 cells (Fig. 4A). However, treated SKOV3 ${ }_{\text {TR }}$ cells showed less effect than SKOV3 cells (Fig. 4B). This may be due to the high P-gp expression in $\mathrm{SKOV}_{\mathrm{TR}}$ cells, which can induce the active excretion of some drugs. However, the proliferation ability of $\mathrm{SKOV}_{\mathrm{TR}}$ cells treated with HA-PTX/miR let-7a-pGNR@MSN was significantly lower than that of SKOV3 $3_{\mathrm{TR}}$ cells treated with PTX/miR let-7a-pGNR@MSN (Fig. 4C). In addition, the HA-PTX/miR let-7a-GNR@MSN nano co-delivery system enhanced therapeutic efficacy, which can be attributed to the target ability of hyaluronic acid to the highly expressed CD44 protein receptor on the surface of SKOV3/SKOV3 ${ }_{\text {TR }}$ cells. (Fig. 4C). As shown in Fig. 4D, E, FDA/PI double-staining analysis showed that HA-PTX/ let-7a-pGNR@MSN had a better therapeutic effect than PTX/let-7a-pGNR@MSN.

Western blotting was also used to detect the expression of P-gp in treated $\mathrm{SKOV}_{\mathrm{TR}}$ cells. It can be seen from Fig. 4F that the expression of P-gp in the HA-miR let7a-pGNR@MSN group was lower than that in the HAPTX-pGNR@MSN group, indicating that let-7a could effectively reduce the expression of $\mathrm{P}$-gp, thus further reducing the drug resistance of SKOV3 $3_{\mathrm{TR}}$ cells to PTX. In addition, the expression of P-gp in the HA-PTX/miR let7a-pGNR@MSN group was the lowest, indicating that 


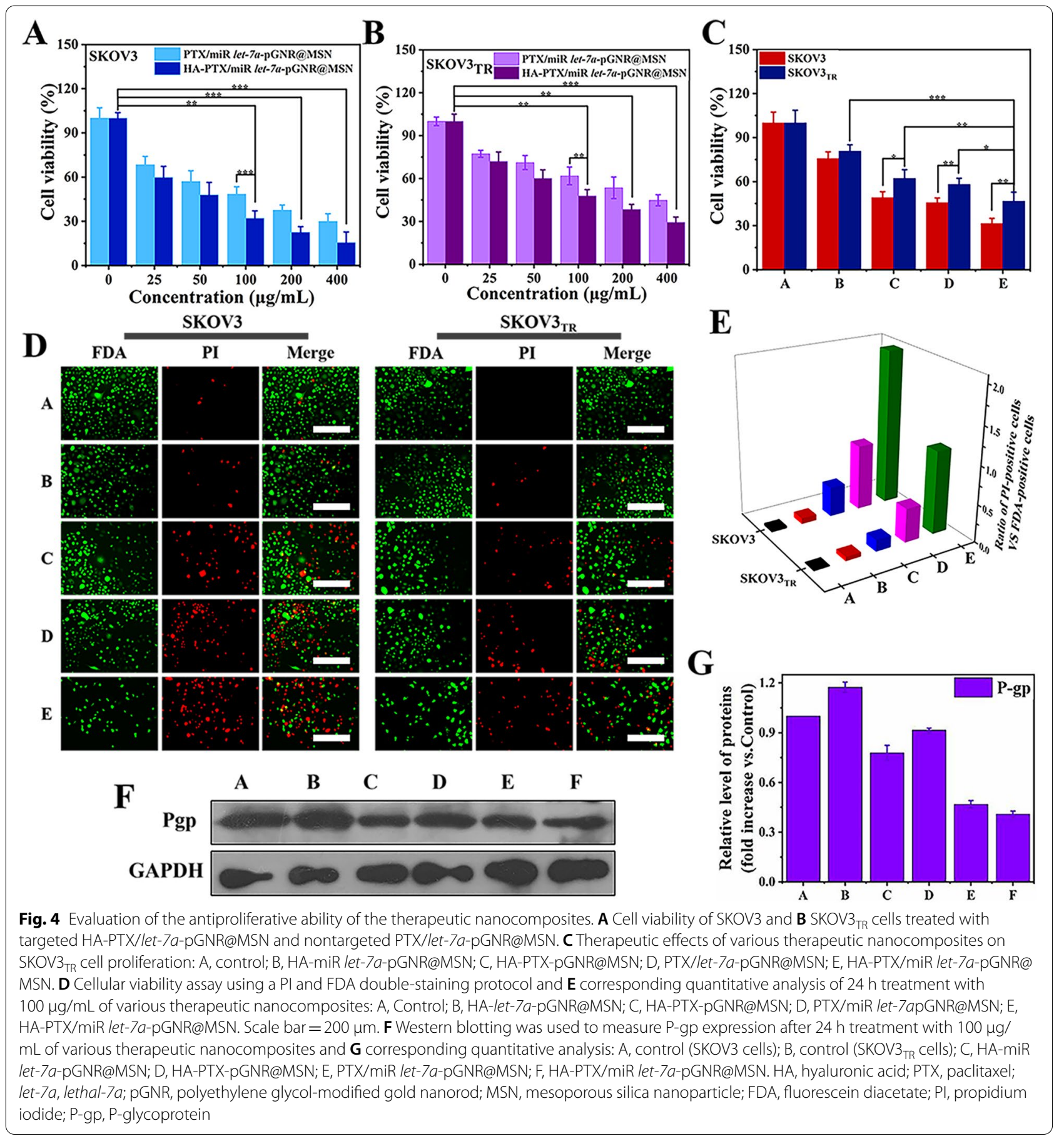

miR let-7a further enhanced the chemotherapy effect of PTX and promoted the apoptosis of SKOV3 ${ }_{\mathrm{TR}}$ cells. As shown in Additional file 1: Fig. S4A, B, the expression of P-gp decreased significantly with the increase of HAPTX/miR let-7a-pGNR@MSN concentration, mainly because the therapeutic nanocomposites reversed MDR in $\mathrm{SKOV}_{\mathrm{TR}}$ cells.

\section{Analysis of the apoptosis mechanism}

In order to observe the nuclear division of apoptotic cells, treated SKOV3 and SKOV3 $3_{\mathrm{TR}}$ cells were stained with Hoechst H33258, a fluorescent dye that binds to ATrich DNA regions [30]. The typical changes in SKOV3 and $\mathrm{SKOV}_{\mathrm{TR}}$ apoptotic cells after treatment were 
chromatin condensation, perinuclear aggregation, and nuclear fragmentation (Fig. 5A, B). In addition, nuclear fragmentation and chromatin condensation were significantly increased with the increase of HA-PTX/miR let7a-pGNR@MSN concentration (Additional file 1: Fig. S5 and Fig. 5C).

Myeloid leukemia factor-1 (Mcl-1), an anti-apoptotic member of the B-cell lymphoma 2 (Bcl-2) family, is highly expressed in cancer cells [36]. The expression of Mcl-1 not only promotes the generation and development of tumors, but also leads to the drug resistance of cancer cells to chemotherapeutic drugs. As shown in Fig. 5D, E, the expression of in the HA-PTX/miR let-7apGNR@MSN group was significantly reduced, indicating that the sensitivity of $\mathrm{SKOV}_{\mathrm{TR}}$ cells were significantly more sensitive to PTX and further induced apoptosis. The expression of Mcl-1 also decreased with the increase of HA-PTX/miR let-7a-pGNR@MSN concentration (Additional file 1: Fig. S6A, B). In addition, the expression of pro-apoptotic Bcl-2-associated X protein (Bax) was increased in SKOV3 ${ }_{\mathrm{TR}}$ cells in the HA-PTX/miR let7a-pGNR@MSN group compared to other groups, while the expression of anti-apoptotic B-cell lymphoma extra large (Bcl-XL) was decreased, indicating that MDR in $\mathrm{SKOV}_{\mathrm{TR}}$ cells can be reversed.

Annexin V-FITC and PI staining were used to further detect the mechanism of MDR reversal. According to fluorescence intensity, the treated cells were divided into four quadrants: living cells, early apoptotic cells, late apoptotic cells and necrotic cells (Fig. 5F). Figure 5G, H show show a quantitative analysis of the number of cells in the four quadrants of each group. The survival rates of SKOV3 and SKOV3 ${ }_{\mathrm{TR}}$ cells were $29.1 \%$ and $42.8 \%$ after treatment with HA-PTX/miR let-7a-pGNR@MSN for $24 \mathrm{~h}$, respectively, indicating that $\mathrm{HA}-\mathrm{PTX} / \mathrm{miR}$ let-7apGNR@MSN significantly affected cell apoptosis. In addition, the number of early apoptotic cells was significantly higher than that of late apoptotic cells, which may be due to the reversal of phosphatidylserine (PS) from the interior to the surface of the cell membrane and exposure to the extracellular environment.

\section{Effects of therapeutic nanocomposites on related} organelles and mTOR-mediated signaling pathways

To determine the effects of various therapeutic nanocomposites on vesicles, SKOV3 and $\mathrm{SKOV}_{\mathrm{TR}}$ cells were stained with $\mathrm{AO}$. Compared to the other groups, a significant increase in the number of induced red fluorescent spots in the cytoplasm was observed in the HA-PTX/miR let-7a-pGNR@MSN group, indicating a decrease in acidic compartments such as lysosomes and autophagic lysosomes (Fig. 6A).

Electrochemical potential energy is stored in the inner membrane of mitochondria. If there is an asymmetric distribution of proton plasma concentration on both sides of the membrane, the mitochondrial membrane potential shows an upward trend, which is called “MMP”. HA-PTX/miR let-7a-pGNR@MSN an increase in intracellular green fluorescent aggregates, suggesting depolarization of mitochondrial intima (Fig. 6B, C). The depolarization process of MMP will cause the mitochondria to release a large amount of cytochrome $\mathrm{c}$ into the cytoplasm, thus accelerating the process of apoptosis. In contrast, intracellular ROS levels were significantly elevated, consistent with earlier results (Fig. 6D, E).

Mammalian target of rapamycin (mTOR) is an important regulator of cell growth and proliferation [37-39]. Abnormal regulation of the mTOR signaling pathway is closely related to cell proliferation. Signal transducers and transcription activators (STAT) are a highly conserved family of transcription factors that are stimulated by extracellular signals for pure phosphorylation and translocation in the cytoplasm. STAT3 is one of the seven members of the STAT family and is involved in cell cycle, apoptosis regulation, tumor angiogenesis, tumor cell invasion, metastasis, and immune escape [40, 41]. Enhancer of zeste homolog 2 (EZH2), which is highly expressed in tumor-resistant cells and has histone methyltransferase activity, is involved in X chromosome inactivation, cell differentiation, and regulation of embryo development [42].

\footnotetext{
(See figure on next page.)

Fig. 5 Apoptosis analysis of treated SKOV3 and SKOV ${ }_{T R}$ cells. A Fluorescence images using Hoechst $\mathrm{H} 33258$ staining and $\mathbf{B}$ corresponding quantitative analysis of apoptotic rate of SKOV3 and SKOV3 ${ }_{T R}$ cells treated with various therapeutic nanocomposites: $A$, control; B, HA-miR let-7a-pGNR@MSN; C, HA-PTX-pGNR @ MSN; D, PTX/miR let-7a pGNR@MSN; E, HA-PTX/miR let-7a-pGNR@MSN. Scale bar=100 $\mu$ m. C Analysis of apoptotic rate of SKOV3 and SKOV3 TR $_{\text {C }}$ cells treated with various concentrations of HA-PTX/miR let-7a-pGNR@MSN (0, 25, 50, 100, 200, and 400 $\mu \mathrm{g} /$ $\mathrm{mL}$ ). D Western blotting of apoptosis-associated proteins in SKOV3 ${ }_{\mathrm{TR}}$ cells treated with various therapeutic nanocomposites and $\mathbf{E}$ statistical analysis of relative protein levels: A, control; B, HA-miR let-7a-pGNR@MSN; C, HA-PTX-pGNR@MSN; D, PTX/miR let-7a pGNR@MSN; E, HA-PTX/ miR let-7a-pGNR@MSN. F Flow cytometric analysis of apoptosis using annexin V-FITC/PI staining of SKOV3 and SKOV3 ${ }_{\text {TR }}$ cells after treatment with various therapeutic nanocomposites. A, control; B, HA-miR let-7a-pGNR@MSN; C, HA-PTX-pGNR@MSN; D, PTX/miR let-7a pGNR@MSN; E, HA-PTX/ miR let-7a-pGNR@MSN. G, H Corresponding quantification analyses of the percentage of live, early apoptotic, late apoptotic, and necrotic cells after treatment of SKOV3 and SKOV3 ${ }_{\text {TR }}$ with various therapeutic nanocomposites: A, control; B, HA-miR let-7a-pGNR@MSN; C, HA-PTX-pGNR@MSN; D, PTX/miR let-7a-pGNR @MSN; E, HA-PTX/miR let-7a-pGNR@MSN. HA, hyaluronic acid; let-7a, lethal-7a; pGNR, polyethylene glycol-modified gold nanorod; MSN, mesoporous silica nanoparticle; PTX, paclitaxel; FITC, fluorescein isothiocyanate; Pl, propidium iodide
} 


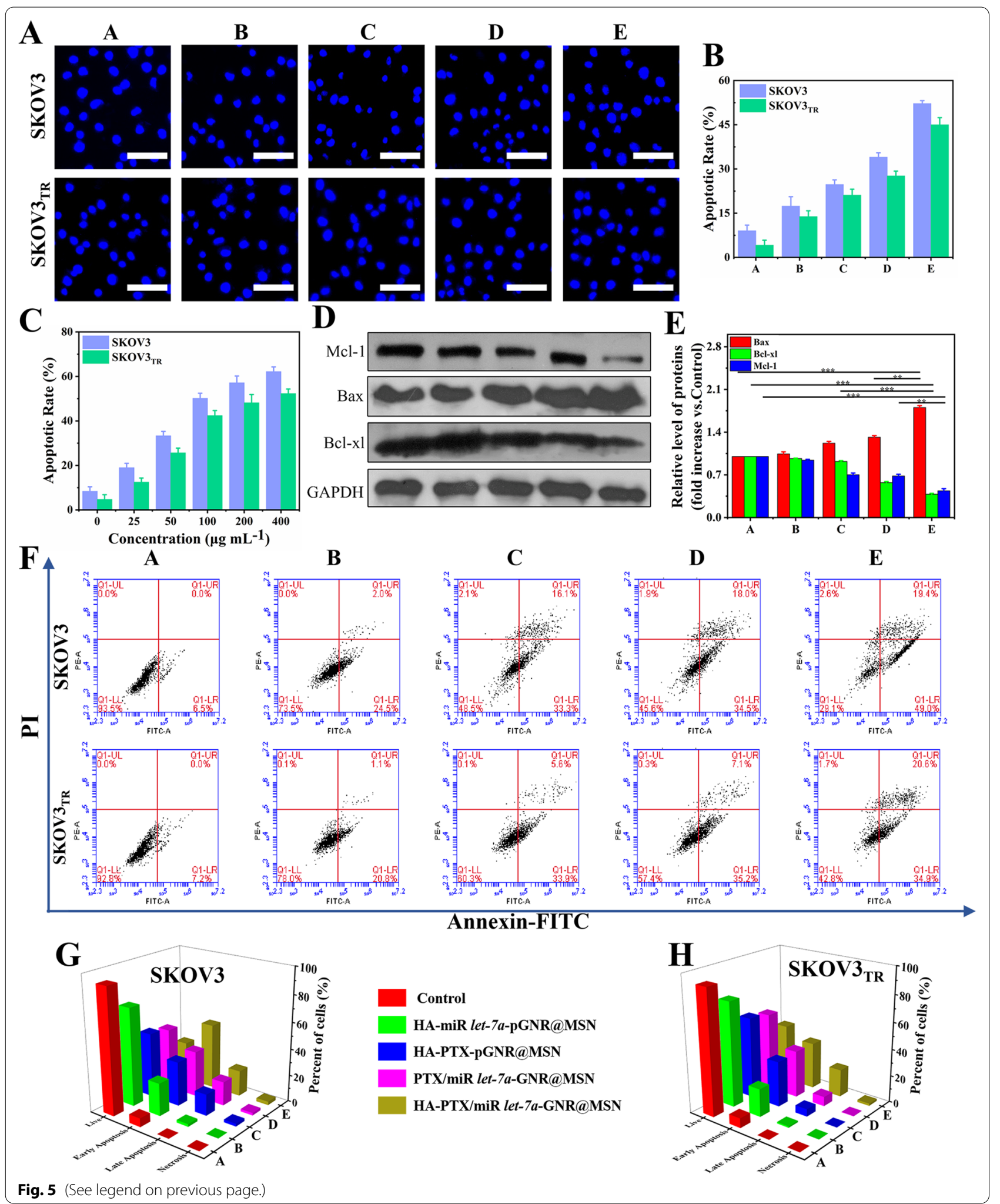




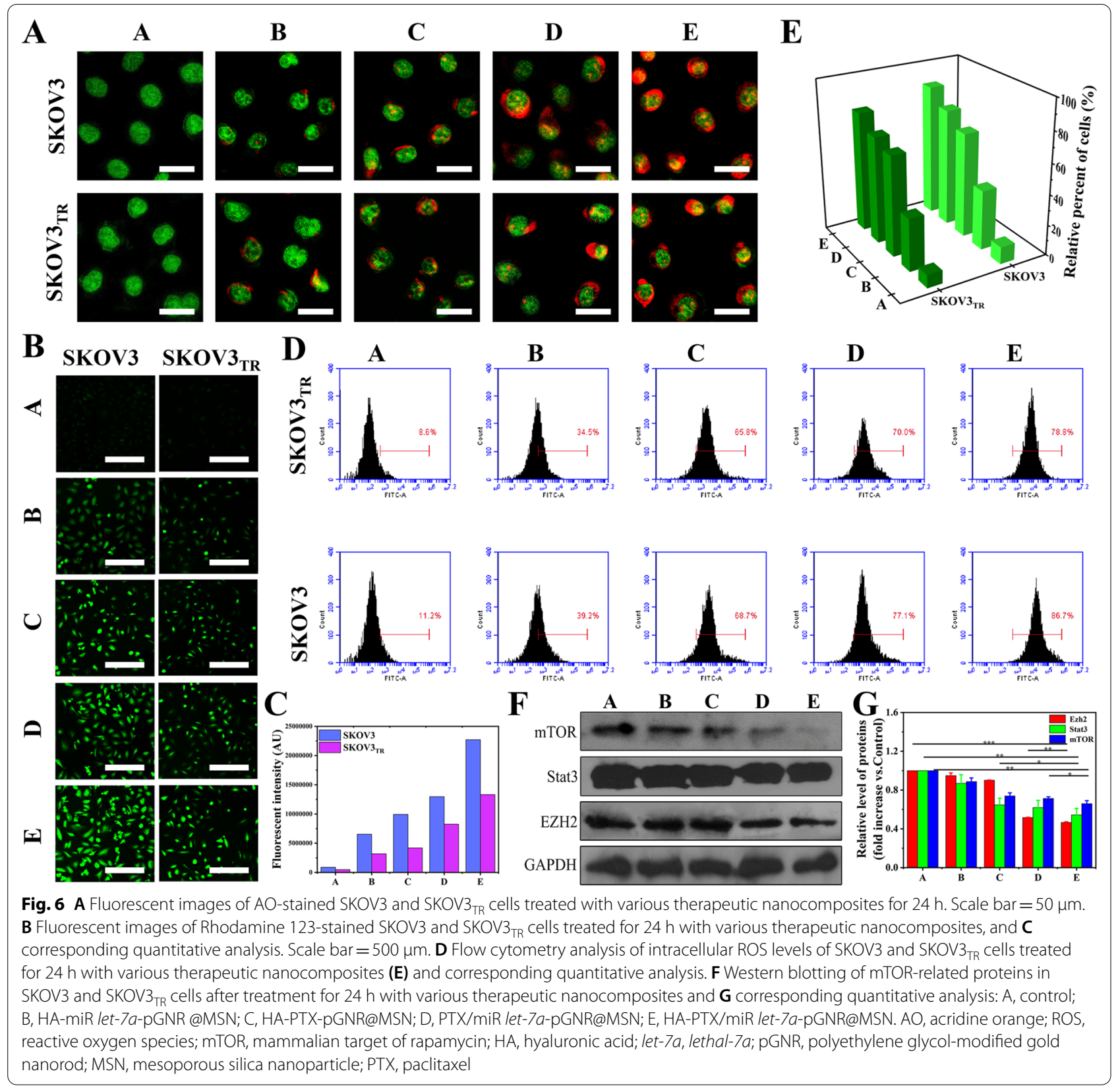

In this study, it was found that the decreased mTORC1 expression leads to a rapid decline in STAT3 level and a decrease in the protein phosphorylation it regulates, thereby reducing the histone methyltransferase activity of EZH2. These cascade reactions may be strong evidence that HA-PTX/miR let-7a-pGNR@MSN reverses $\mathrm{MDR}$ and inhibits the proliferation of $\mathrm{SKOV}_{\mathrm{TR}}$ cells (Fig. 6F, G).

\section{Reversal of MDR in ovarian cancer in vivo}

HA-PTX/miR let-7a-pGNR@MSN showed favorable ability of reversing MDR in vitro. Therefore, SPF $\mathrm{SKOV}_{\mathrm{TR}}$ BALB/c-nu mice were selected to establish a subcutaneous tumor model and treated with various therapeutic nanocomposites $(15 \mathrm{mg} / \mathrm{kg}$ ) every 2 days (Fig. 7A). After 15 days of treatment, the mice were euthanized and their main organs and tumor tissues were collected. As shown 

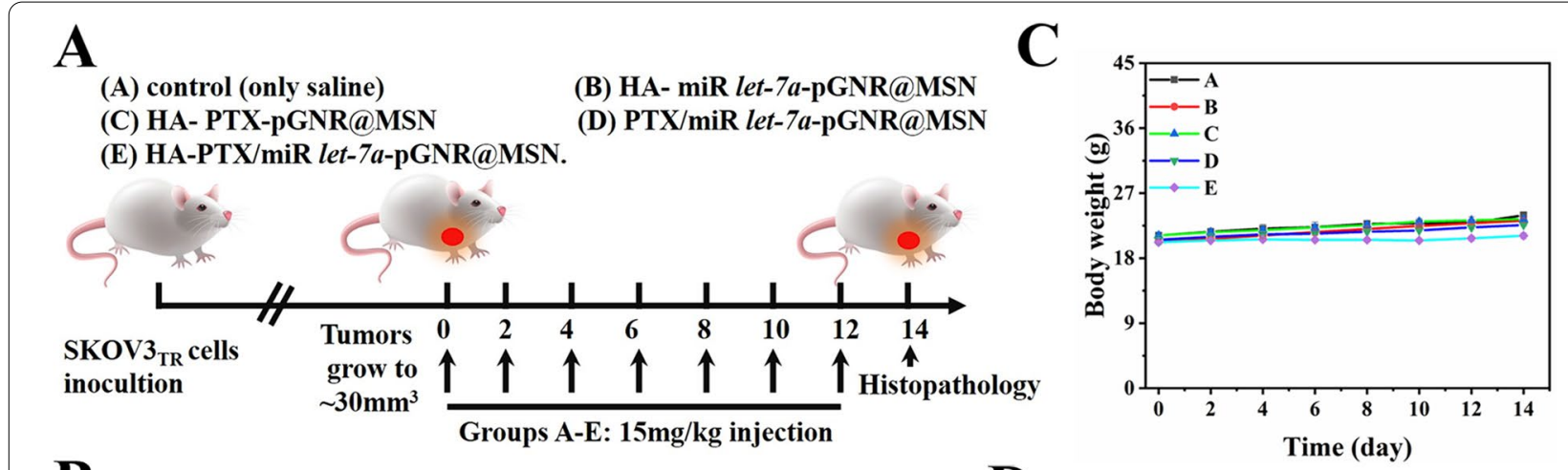

B

(A)

(B)

(C)

(D)

(E)

D
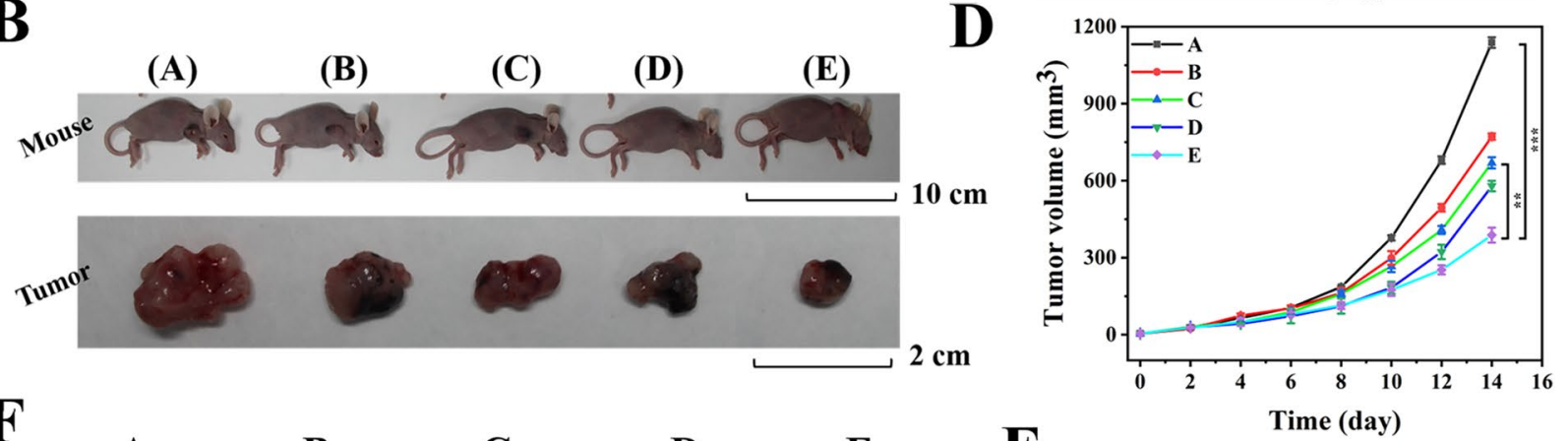

F

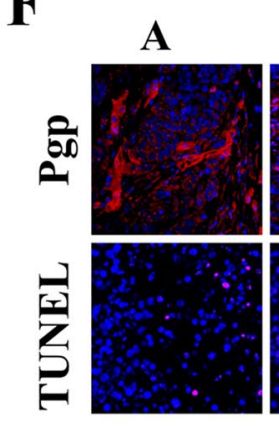

B

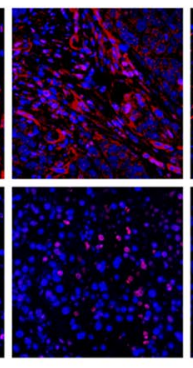

A

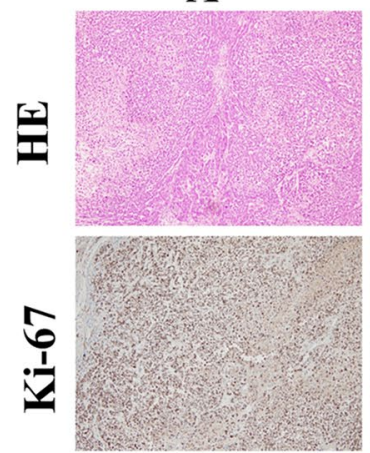

C

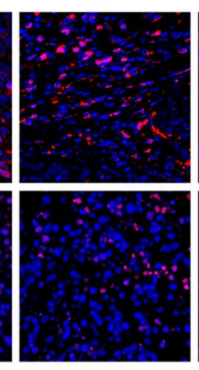

B

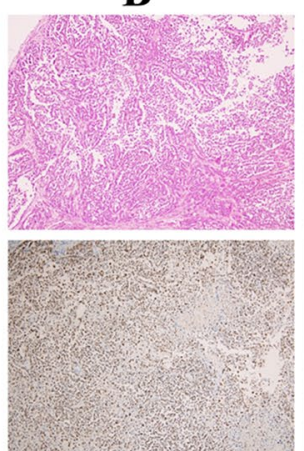

D
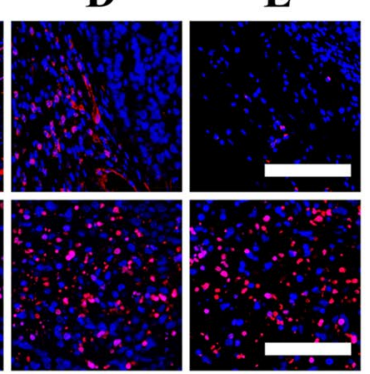

C

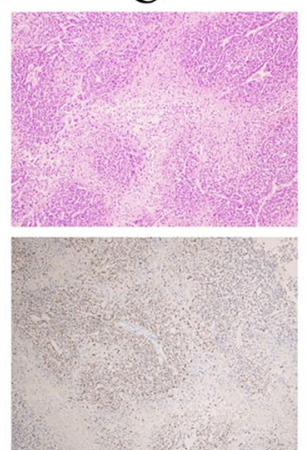

$\mathbf{E}$

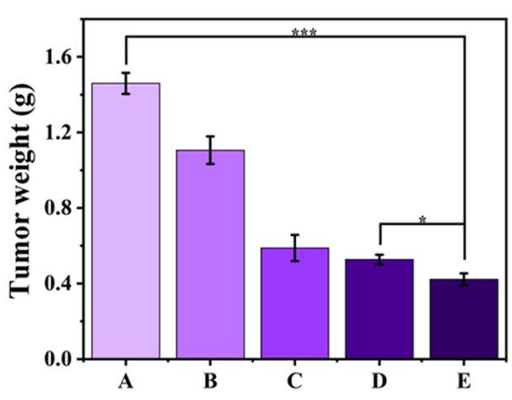

D

E
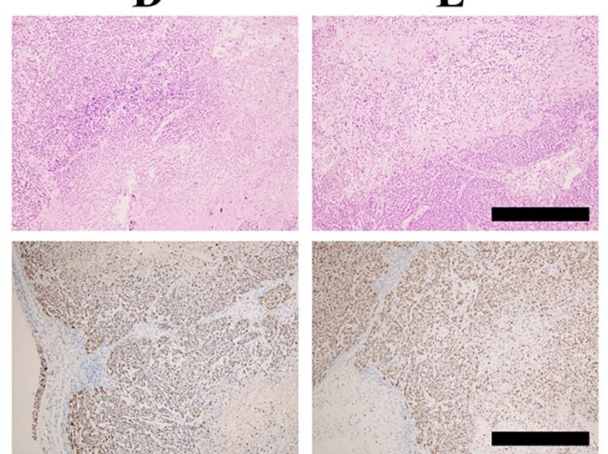

Fig. 7 Therapeutic efficacy of various nanocomposites in vivo. A Experimental procedure designed for nanocomposite therapy. B Body weight curve of mice after intratumoral injection with various therapeutic nanocomposites following the experimental scheme. C Digital photographs of mice and dissected tumors. D Time-dependent tumor growth curves $(n=5$, mean \pm SD). E Tumor weight after 15 days of treatment. $\mathbf{F}$ P-gp immunohistochemical staining and TUNEL staining for analysis of pathological changes. Scale bar $=100 \mu \mathrm{m}$. G H\&E staining and Ki-67 immunohistochemical staining for cellular proliferation analysis in tumor tissue after 15 days of treatment. Scale bar $=500 \mu m$. NP, nanoparticle; SD, standard deviation; TUNEL, terminal deoxynucleotidyl transferase dUTP nick end labeling; P-gp, P-glycoprotein; H\&E, hematoxylin and eosin 
in Fig. 7B, there was no significant change in the body weight of all the mice, indicating that HA-pGNR@MSN nanocarriers had no significant toxicity to mice during the treatment. It was also shown that the HA-PTX/miR let7a-pGNR@MSN group demonstrated better therapeutic effect compared to the PTX/miR let-7a-pGNR@MSN group. In addition, co-delivery of $\mathrm{PTX} / \mathrm{miR}$ using HApGNR@MSN as nanocarriers effectively inhibited the growth of $\mathrm{SKOV}_{\mathrm{TR}}$ after intratumoral administration, which had a synergistic inhibitory effect on tumor growth (Fig. 7C-E).

The mechanism was further analyzed using antigen P-gp and TUNEL staining assay. After treatment with HA-PTX/miR let-7a-pGNR@MSN, the level of P-gp in the cancer tissue significantly decreased, which was consistent with in vitro results (Fig. 7F). TUNEL staining results showed that the staining signal decreased, indicating that the apoptosis of cancer cells of the HA-PTX/ miR let-7a-pGNR@MSN group increased compared to other groups (Fig. 7F). H\&E staining assay clearly showed significant destruction of solid tumors in the HA-PTX/ miR let-7a-pGNR@MSN group (Fig. 7G). The decrease of Ki-67 level in the tumor tissue also indicated that the proliferation ability and malignancy of tumors decreased after treatment with HA-PTX/miR let-7a-pGNR@MSN (Fig. 7G). In addition, H\&E staining showed the complete structure of major organs and no obvious tumor invasion and metastasis, indicating that $\mathrm{HA}-\mathrm{PTX} / \mathrm{miR}$ let-7apGNR@MSN had no obvious damage to major organs, further indicating that the nanoparticles had low toxicity and good safety (Fig. 8).

\section{Conclusions}

In summary, we successfully developed a novel HA-modified targeted nanosystem pGNR@MSN for co-delivery of miR let-7a and PTX to overcome MDR in ovarian cancer and enhance chemotherapy effect. Our experimental results demonstrated that PTX and miR let-7/PTX could be effectively co-delivered to SKOV3/SKOV $3_{\mathrm{TR}}$ cells and ovarian cancer tissue by this nanosystem. Moreover, miR let-7a decreased P-gp expression, reversed the MDR of SKOV $3_{\mathrm{TR}}$ cells and ovarian cancer tissue, and enhanced the therapeutic effect of PTX, leading to high therapeutic efficiency. In the mantime, in vivo experiments showed that the delivery system had almost no toxicity. In-depth analysis of the experimental data implied that mTORmediated signaling pathways played an important role in reversing drug resistance and subsequent inducting

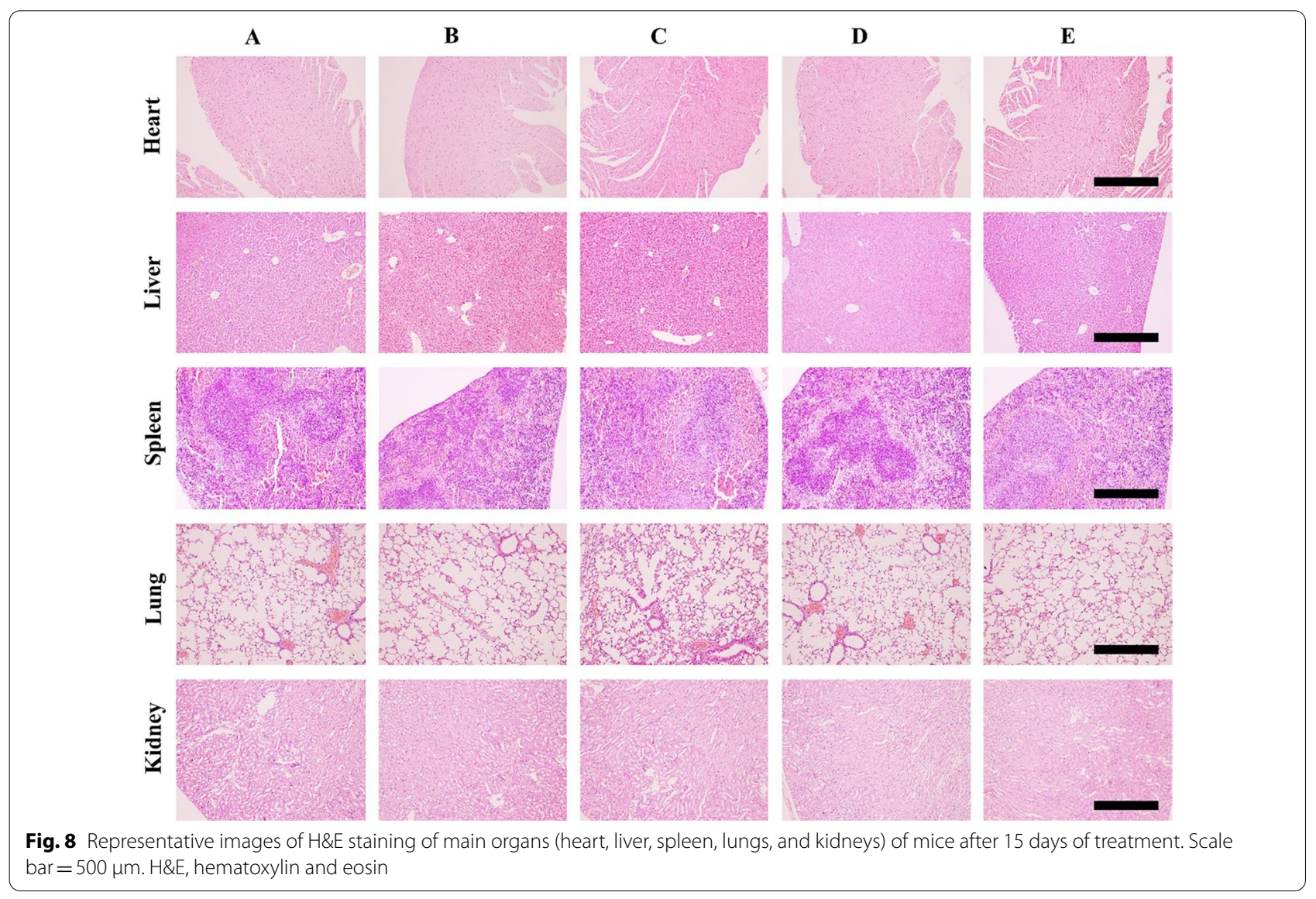


apoptosis. Hence, this study shall provide theoretical guidance and effective basis for treatment of ovarian cancer in the future.

\begin{abstract}
Abbreviations
MDR: Multidrug resistance; P-gp: P-glycoprotein; miR: MicroRNA; let-7a: lethal7a; PTX: Paclitaxel; HA: Hyaluronic acid; GNR: Gold nanorods; ATP: Adenosine triphosphate; MSN: Mesoporous silica nanoparticle; PEG: Polyethylene glycol; pGNR: Polyethylene glycol-modified gold nanorods; TEOS: Tetraethyl orthosilicate; FBS: Fetal bovine serum; RPMI: Roswell Park Memorial Institute; AO: Acridine orange; FDA: Fluorescein diacetate; PI: Propidium iodide; DAPI: 4',6-Diamidino-2-phenylindole; MTT: 3-(4,5-Dimethylthiazlo-2-diphenyltetrazolium) bromide; RBITC: Rhodamine B isothiocyanate; DMSO: Dimethyl sulfoxide; FITC: Fluorescein isothiocyanate; CTAB: Cetyltrimethylammonium bromide; DI: Deionized; APTES: 3-Aminopropyltriethoxysilane; HPLC: Highperformance liquid chromatography; EDC: 1-Ethyl-3-(3-dimethylaminopropyl) carbodiimide hydrochloride; NHS: N-Hydroxysuccinimide; MES: 2-(N-Morpholino)ethanesulfonic acid; RT: Room temperature; HAADF-STEM: High-angle annular dark-field scanning transmission electron microscopy; HRTEM: High-resolution transmission electron microscopy; DLS: Dynamic laser scattering; FT-IR: Fourier transform infrared; XRD: X-ray diffraction; PBS: Phosphatebuffered saline; FAM: Carboxyfluorescein; MMP: Mitochondrial membrane potential; ROS: Reactive oxygen species; DCFH-DA: 2'-7’Dichlorofluorescin diacetate; SDS: Sodium dodecyl sulfate; PMSF: Phenylmethylsulfonyl fluoride; SDS-PAGE: Sodium dodecyl sulfate-polyacrylamide gel electrophoresis; PVDF: Polyvinylidene difluoride; PBST: Phosphate-buffered saline-Tween 20; HRP: Horseradish peroxidase; GADPH: Glyceraldehyde 3-phosphate dehydrogenase; SPF: Specific pathogen-free; H\&E: Hematoxylin and eosin; TUNEL: Terminal deoxynucleotidyl transferase dUTP nick end labeling; EDS: Energy-dispersive X-ray spectroscopy; SSA: Specific surface area; RBC: Red blood cell; TEM: Transmission electron microscopy; BCl-2: B-cell lymphoma 2; Mcl-1: Myeloid leukemia factor-1; Bax: Bcl-2-associated X protein; Bcl-XL: B-cell lymphoma extra large; PS: Phosphatidylserine; mTOR: Mammalian target of rapamycin; STAT: Signal transducer and activator of transcription; NP: Nanoparticle; SD: Standard deviation.
\end{abstract}

\section{Supplementary Information}

The online version contains supplementary material available at https://doi. org/10.1186/s12951-021-01139-1.

Additional file 1. Additional Figures S1-S6.

\section{Authors' contributions}

YG and XW designed the study. TX, MC, NL, QL and LZ performed experiments and data analysis. XW, TX and MC wrote the manuscript. SD and YW revised the manuscript. All authors read and approved the final manuscript.

\section{Funding}

This work was kindly supported by the National Natural Science Foundation of China (No. 815725 74), China Postdoctoral Science Foundation (No. 2019M652541), Innovation Scientists and Technicians Troop Construction Projects of Henan Province (No. 192101510001), the Young Core Instructor Program in Higher Education Institution of Henan province (No. 2018 GGJS 067), Henan Province medical science and technology research project jointly built by the Ministry and province (No. SB201901073), and the Basic Research Project of Key Scientific Research Projects in Henan Province (No. 20zx011).

\section{Availability of data and materials}

All data generated or analyzed during this study are included in this published article (and also in Additional information).

\section{Declarations}

Ethics approval and consent to participate

Animal experiments were conducted according to the guidelines specified by the Animal Care and Use Committee of Zhengzhou University (Zhengzhou, China) (Zhengzhou, China).

\section{Consent for publication}

Not applicable.

\section{Competing interests}

The authors declare no conflict of interest.

\section{Author details}

${ }^{1}$ Henan Provincial People's Hospital, Zhengzhou 450003, China. ${ }^{2}$ College of Bioengineering, Henan University of Technology, Zhengzhou 450001, China. ${ }^{3}$ People's Hospital of Zhengzhou University, Zhengzhou 450003, China. ${ }^{4}$ Henan International Joint Laboratory for Gynecological Oncology and Nanomedicine, Zhengzhou 450003, China. ${ }^{5}$ Institute for Innovative Drug Design and Evaluation, School of Pharmacy, Henan University, Kaifeng 475004, China. ${ }^{6}$ Henan International Joint Laboratory of Chinese Medicine Efficacy, Henan University, Kaifeng 475004, China. ${ }^{7}$ Henan Bioengineering Research Center, Zhengzhou 450046, China.

Received: 30 August 2021 Accepted: 15 November 2021

Published online: 23 December 2021

\section{References}

1. Lheureux S, Braunstein M, Oza AM. Epithelial ovarian cancer: evolution of management in the era of precision medicine. CA Cancer J Clin. 2019;69:280-304.

2. Shi T, Zhu J, Feng Y, Tu D, Zhang Y, Zhang P, et al. Secondary cytoreduction followed by chemotherapy versus chemotherapy alone in platinumsensitive relapsed ovarian cancer (SOC-1): a multicentre, open-label, randomised, phase 3 trial. Lancet Oncol. 2021;22:439-49.

3. Baekelandt M, Lehne G, Trope CG, Szanto I, Pfeiffer P, Gustavssson B, et al. Phase I/II trial of the multidrug-resistance modulator valspodar combined with cisplatin and doxorubicin in refractory ovarian cancer. J Clin Oncol. 2001;19:2983-93.

4. He C, Poon C, Chan C, Yamada SD, Lin W. Nanoscale coordination polymers codeliver chemotherapeutics and siRNAs to eradicate tumors of cisplatin-resistant ovarian cancer. J Am Chem Soc. 2016;138:6010-9.

5. Johnatty SE, Beesley J, Paul J, Fereday S, Spurdle AB, Webb PM, et al. ABCB1 (MDR 1) polymorphisms and progression-free survival among women with ovarian cancer following paclitaxel/carboplatin chemotherapy. Clin Cancer Res. 2008;14:5594-601.

6. Bookman MA, Gilks CB, Kohn EC, Kaplan KO, Huntsman D, Aghajanian $C$, et al. Better therapeutic trials in ovarian cancer. J Natl Cancer Inst. 2014;106:1-8.

7. Patch AM, Christie EL, Etemadmoghadam D, Garsed DW, George J, Fereday $\mathrm{S}$, et al. Whole-genome characterization of chemoresistant ovarian cancer. Nature. 2015:521:489-94.

8. Badmann S, Heublein S, Mayr D, Reischer A, Liao Y, Kolben T, et al. M2 macrophages infiltrating epithelial ovarian cancer express MDR1: a feature that may account for the poor prognosis. Cells. 2020;9:1-14.

9. Zhang R, Gao S, Wang Z, Han D, Liu L, Ma Q, et al. Multifunctional molecular beacon micelles for intracellular mRNA imaging and synergistic therapy in multidrug-resistant cancer cells. Adv Funct Mater. 2017;27:1701027

10. Szakacs G, Paterson JK, Ludwig JA, Booth-Genthe C, Gottesman MM. Targeting multidrug resistance in cancer. Nat Rev Drug Discov. 2006;5:219-34

11. Mezzanzanica D, Bagnoli M, De Cecco L, Valeri B, Canevari S. Role of microRNAs in ovarian cancer pathogenesis and potential clinical implications. Int J Biochem Cell Biol. 2010;42:1262-72.

12. Liu WM, Lv CP, Zhang B, Zhou QS, Cao ZF. MicroRNA-27b functions as a new inhibitor of ovarian cancer-mediated vasculogenic mimicry through suppression of VE-cadherin expression. RNA. 2017;23:1019-27. 
13. Mihanfar A, Fattahi A, Nejabati HR. MicroRNA-mediated drug resistance in ovarian cancer. J Cell Physiol. 2019;234:3180-91.

14. Boyerinas B, Park SM, Hau A, Murmann AE, Peter ME. The role of let-7 in cell differentiation and cancer. Endocr Relat Cancer. 2010;17:F19-36.

15. Mondol V, Pasquinelli AE. Let's make it happen: the role of let-7 microRNA in development. Curr Top Dev Biol. 2012;99:1-30.

16. Boyerinas B, Park S-M, Murmann AE, Gwin K, Montag AG, Zillhardt M, Hua Y-J, Lengyel E, Peter ME. Let-7 modulates acquired resistance of ovarian cancer to Taxanes via IMP-1-mediated stabilization of multidrug resistance 1. Int J Cancer. 2012;130(8):1787-97. https://doi.org/10.1002/ijc. 26190.

17. Wan D, Li C, Pan J. Polymeric micelles with reduction-responsive function for targeted cancer chemotherapy. ACS Appl Biol Mater. 2020;3:1 139-46.

18. Gong K, Jiao JY, Xu CQ, Dong Y, Li DX, He D, Zhao D, Yu J, Sun Y, Zhang W, Bai M, Duan YR. The targetable nanoparticle BAF312@cRGD-CaP-NP represses tumor growth and angiogenesis by downregulating the S1PR1/ P-STAT3/NEGFA axis in triple-negative breast cancer. J Nanobiotechnol. 2021;19:165.

19. Kankala RK, Han YH, Na J, Lee CH, Sun Z, Wang SB, et al. Nanoarchitectured structure and surface biofunctionality of mesoporous silica nanoparticles. Adv Mater. 2020;32:1-27.

20. Kim D, Choi E, Lee C, Choi Y, Kim H, Yu T, et al. Highly sensitive and selective visual detection of $\mathrm{Cr}(\mathrm{VI})$ ions based on etching of silver-coated gold nanorods. Nano Converg. 2019;6:34

21. Chen YS, Zhao Y, Yoon SJ, Gambhir SS, Emelianov S. Miniature gold nanorods for photoacoustic molecular imaging in the second nearinfrared optical window. Nat Nanotechnol. 2019;14:465-72.

22. Liu S, Zhang Y, Zhao X, Wang J, Di C, Zhao Y, et al. Tumor-specific silencing of tissue factor suppresses metastasis and prevents cancer-associated hypercoagulability. Nano Lett. 2019;19:4721-30.

23. Liu Y, Bhattarai P, Dai Z, Chen X. Photothermal therapy and photoacoustic imaging via nanotheranostics in fighting cancer. Chem Soc Rev. 2019:48:2053-108.

24. Wang Y, Zeng Y, Fu W, Zhang P, Li L, Ye C, et al. Seed-mediated growth of Au@Ag core-shell nanorods for the detection of ellagic acid in whitening cosmetics. Anal Chim Acta. 2018;1002:97-104.

25. Bae KH, Tan S, Yamashita A, Ang WX, Gao SJ, Wang S, et al. Hyaluronic acid-green tea catechin micellar nanocomplexes: fail-safe cisplatin nanomedicine for the treatment of ovarian cancer without off-target toxicity. Biomaterials. 2017;148:41-53.

26. Yang X, lyer AK, Singh A, Choy E, Hornicek FJ, Amiji MM, et al. MDR1 siRNA loaded hyaluronic acid-based CD44 targeted nanoparticle systems circumvent paclitaxel resistance in ovarian cancer. Sci Rep. 2015;5:8509.

27. Wang C, Ma Z, Wang T, Su Z. Synthesis, assembly, and biofunctionalization of silica-coated gold nanorods for colorimetric biosensing. Adv Funct Mater. 2006:16:1673-8.

28. Cui Y, Xu Q, Chow PK, Wang D, Wang CH. Transferrin-conjugated magnetic silica PLGA nanoparticles loaded with doxorubicin and paclitaxel for brain glioma treatment. Biomaterials. 2013;34:8511-20.

29. Hou W, Xia F, Alfranca G, Yan H, Zhi X, Liu Y, et al. Nanoparticles for multimodality cancer diagnosis: simple protocol for self-assembly of gold nanoclusters mediated by gadolinium ions. Biomaterials. 2017;120:103-14.

30. Wang $X$, Li R, Zhu Y, Wang Z, Zhang H, Cui L, et al. Active targeting co-delivery of therapeutic Sur siRNA and an antineoplastic drug via epidermal growth factor receptor-mediated magnetic nanoparticles for synergistic programmed cell death in glioblastoma stem cells. Mater Chem Front. 2020:4:574-88.

31. Wang X, Xiong T, Cui M, Guan X, Yuan J, Wang Z, et al. Targeted selfactivating $\mathrm{Au}-\mathrm{Fe} 3 \mathrm{O} 4$ composite nanocatalyst for enhanced precise hepatocellular carcinoma therapy via dual nanozyme-catalyzed cascade reactions. Appl Mater Today. 2020;21:100827.

32. Kamath PR, Sunil D, Joseph MM, Abdul Salam AA, Sreelekha TT. Indolecoumarin-thiadiazole hybrids: an appraisal of their MCF-7 cell growth inhibition, apoptotic, antimetastatic and computational $\mathrm{Bcl}-2$ binding potential. Eur J Med Chem. 2017;136:442-51.

33. Merino D, Kelly GL, Lessene G, Wei AH, Roberts AW, Strasser A. BH3mimetic drugs: blazing the trail for new cancer medicines. Cancer Cell. 2018:34:879-91.

34. Cheng Z, He Z, Cai Y, Zhang C, Fu G, Li H, et al. Conversion of hepatoma cells to hepatocyte-like cells by defined hepatocyte nuclear factors. Cell Res. 2019:29(2):124-35.
35. Lang FF, Conrad C, Gomez MC, Yung W, Sawaya R, Weinberg JS, et al. Phase I study of DNX-2401 (Delta-24-RGD) oncolytic adenovirus: replication and immunotherapeutic effects in recurrent malignant glioma. J Clin Oncol. 2018;36(14):1419-27.

36. Silva MC, Nandi GA, Tentarelli S, Gurrell IK, Jamier T, Lucente D, et al. Prolonged tau clearance and stress vulnerability rescue by pharmacological activation of autophagy in tauopathy neurons. Nat Commun. 2020;11:3258

37. Huang X, Zhang G, Bai X, Liang T. Combinational therapy targeting the MET-mTOR-ROS loop disrupts mitochondrial autoregulatory machinery of liver cancer. Clin Transl Med. 2020;10:e237.

38. Erin N, Grahovac J, Brozovic A, Efferth T. Tumor microenvironment and epithelial mesenchymal transition as targets to overcome tumor multidrug resistance. Drug Resist Updat. 2020;53:100715-95.

39. Kalantzopoulos GN, Lundvall F, Checchia S, Lind A, Wragg DS, Fjellvag H, et al. In situ flow MAS NMR spectroscopy and synchrotron PDF analyses of the local response of the bronsted acidic site in SAPO-34 during hydration at elevated temperatures. ChemPhysChem. 2018;19:519-28.

40. Kumar V, Cheng P, Condamine T, Mony S, Languino LR, McCaffrey JC, et al. CD45 1 phosphatase inhibits STAT3 transcription factor activity in myeloid cells and promotes tumor-associated macrophage differentiation. Immunity. 2016:44:303-15.

41. Dong Z, Gao M, Li C, Xu M, Liu S. LncRNA UCA1 antagonizes arsenicinduced cell cycle arrest through destabilizing EZH2 and facilitating NFATc2 expression. Adv Sci (Weinh). 2020;7:1903630-40.

42. Pastushenko I, Mauri F, Song Y, De CF, Meeusen B, Swedlund B, et al. Fat1 deletion promotes hybrid EMT state, tumour stemness and metastasis. Nature. 2021;589(7842):448-55.

\section{Publisher's Note}

Springer Nature remains neutral with regard to jurisdictional claims in published maps and institutional affiliations.

Ready to submit your research? Choose BMC and benefit from

- fast, convenient online submission

- thorough peer review by experienced researchers in your field

- rapid publication on acceptance

- support for research data, including large and complex data types

- gold Open Access which fosters wider collaboration and increased citations

- maximum visibility for your research: over $100 \mathrm{M}$ website views per year

At BMC, research is always in progress.

Learn more biomedcentral.com/submissions 